TRANSACTIONS OF THE

AMERICAN MATHEMATICAL SOCIETY

Volume 364, Number 3, March 2012, Pages 1109-1126

S 0002-9947(2011)05327-4

Article electronically published on October 13, 2011

\title{
LIPSCHITZ EQUIVALENCE OF CANTOR SETS AND ALGEBRAIC PROPERTIES OF CONTRACTION RATIOS
}

\author{
HUI RAO, HUO-JUN RUAN, AND YANG WANG
}

\begin{abstract}
In this paper we investigate the Lipschitz equivalence of dust-like self-similar sets in $\mathbb{R}^{d}$. One of the fundamental results by Falconer and Marsh [On the Lipschitz equivalence of Cantor sets, Mathematika, 39 (1992), 223233] establishes conditions for Lipschitz equivalence based on the algebraic properties of the contraction ratios of the self-similar sets. In this paper we extend the study by examining deeper such connections.

A key ingredient of our study is the introduction of a new equivalent relation between two dust-like self-similar sets called a matchable condition. Thanks to a certain measure-preserving property of bi-Lipschitz maps between dust-like self-similar sets, we show that the matchable condition is a necessary condition for Lipschitz equivalence.

Using the matchable condition we prove several conditions on the Lipschitz equivalence of dust-like self-similar sets based on the algebraic properties of the contraction ratios, which include a complete characterization of Lipschitz equivalence when the multiplication groups generated by the contraction ratios have full rank. We also completely characterize the Lipschitz equivalence of dust-like self-similar sets with two branches (i.e., they are generated by IFS with two contractive similarities). Some other results are also presented, including a complete characterization of Lipschitz equivalence when one of the self-similar sets has uniform contraction ratio.
\end{abstract}

\section{INTRODUCTION}

Let $E, F$ be compact sets in $\mathbb{R}^{d}$. We say that $E$ and $F$ are Lipschitz equivalent, and denote it by $E \sim F$, if there exists a bijection $\psi: E \longrightarrow F$ which is bi-Lipschitz, i.e., there exists a constant $C>0$ such that

$$
C^{-1}|x-y| \leq|\psi(x)-\psi(y)| \leq C|x-y|
$$

for all $x, y \in E$.

Received by the editors December 8, 2009.

2010 Mathematics Subject Classification. Primary 28A80.

Key words and phrases. Lipschitz equivalence, dust-like self-similar sets, matchable condition, algebraic rank, uniform contraction ratio.

The research of the first author was supported by the NSFC grants 10971013 and 11171128 .

The research of the second author, who is the corresponding author, was supported in part by the NSFC grant 10601049 and by the Future Academic Star project of Zhejiang University.

The research of the third author was supported in part by NSF Grant DMS-0813750.

(C)2011 American Mathematical Society Reverts to public domain 28 years from publication 
An area of interest in the study of self-similar sets is the Lipschitz equivalence property. With Lipschitz equivalence many important properties of a self-similar set are preserved. Cooper and Pignataro [1] studied the case when $E, F \subset[0,1]$ and $\psi$ is order-preserving. Falconer and Marsh [5, 6] studied quasi-circles and dust-like self-similar sets. In the book of David and Semmes [2, several problems concerning the Lipschitz equivalence of non-dust-like self-similar sets were posed. Using graph-directed sets, Rao, Ruan and $\mathrm{Xi}$ 11] solved one of the problems, the so-called $\{1,3,5\}-\{1,4,5\}$ problem 1 some generalizations were made in [19, 17. For related works on Lipschitz equivalence of other fractals, see [10, 12, 14, 16].

This paper concerns the Lipschitz equivalence of dust-like self-similar sets in $\mathbb{R}^{d}$. Recall that in general we characterize a self-similar set as the attractor of an iterated functions system (IFS). Let $\left\{\phi_{j}\right\}_{j=1}^{m}$ be an IFS on $\mathbb{R}^{d}$ where each $\phi_{j}$ is a contractive similarity with contraction ratio $0<\rho_{j}<1$. The attractor of the IFS is the unique non-empty compact set $F$ satisfying $F=\bigcup_{j=1}^{m} \phi_{j}(F)$; see [8]. We say that the attractor $F$ is dust-like or, alternatively, the IFS $\left\{\phi_{j}\right\}$ satisfies the strong separation condition (SSC) if the sets $\left\{\phi_{j}(F)\right\}$ are disjoint. It is well known that if $F$ is dust-like, then the Hausdorff dimension $s=\operatorname{dim}_{H}(F)$ of $F$ satisfies $\sum_{j=1}^{m} \rho_{j}^{s}=1$.

Now for any $\rho_{1}, \ldots, \rho_{m} \in(0,1)$ with $\sum_{j=1}^{m} \rho_{j}^{d}<1$, we will call $\boldsymbol{\rho}=\left(\rho_{1}, \ldots, \rho_{m}\right)$ a contraction vector and use the notation $\mathcal{D}(\boldsymbol{\rho})=\mathcal{D}\left(\rho_{1}, \ldots, \rho_{m}\right)$ to denote the set of all dust-like self-similar sets that are the attractor of some IFS with contraction ratios $\rho_{j}, j=1, \ldots, m$, on $\mathbb{R}^{d}$. (Throughout the paper the dimension $d$ will be implicit.) Clearly all sets in $\mathcal{D}(\boldsymbol{\rho})$ have the same Hausdorff dimension, which we denote by $s=\operatorname{dim}_{H} \mathcal{D}(\boldsymbol{\rho})$. We are less concerned with the translation part of the IFS's because of the following result; see e.g. 11]:

Proposition 1.1. Let $E, F \in \mathcal{D}\left(\rho_{1}, \ldots, \rho_{m}\right)$. Then $E$ and $F$ are Lipschitz equivalent.

Let $\boldsymbol{\rho}=\left(\rho_{1}, \ldots, \rho_{m}\right)$ and $\boldsymbol{\tau}=\left(\tau_{1}, \ldots, \tau_{n}\right)$ be two contraction vectors. According to Proposition 1.1, we give the following definition: We say $\mathcal{D}(\boldsymbol{\rho})$ and $\mathcal{D}(\boldsymbol{\tau})$ are Lipschitz equivalent, and denote it by $\mathcal{D}(\boldsymbol{\rho}) \sim \mathcal{D}(\boldsymbol{\tau})$, if $E \sim F$ for some (and thus for all) $E \in \mathcal{D}(\boldsymbol{\rho})$ and $F \in \mathcal{D}(\boldsymbol{\tau})$. Note that if $\boldsymbol{\tau}$ is a permutation of $\boldsymbol{\rho}$, then we clearly have $\mathcal{D}(\boldsymbol{\tau})=\mathcal{D}(\boldsymbol{\rho})$. One of the most fundamental results in the study of Lipschitz equivalence is the following theorem, proved by Falconer and Marsh [6], that establishes a connection to the algebraic properties of the contraction ratios:

Theorem 1.2 ([6], Theorem 3.3). Let $\mathcal{D}(\boldsymbol{\rho})$ and $\mathcal{D}(\boldsymbol{\tau})$ be Lipschitz equivalent, where $\boldsymbol{\rho}=\left(\rho_{1}, \ldots, \rho_{m}\right)$ and $\boldsymbol{\tau}=\left(\tau_{1}, \ldots, \tau_{n}\right)$ are two contraction vectors. Let $s=\operatorname{dim}_{H} \mathcal{D}(\boldsymbol{\rho})=\operatorname{dim}_{H} \mathcal{D}(\boldsymbol{\tau})$. Then:

(1) $\mathbb{Q}\left(\rho_{1}^{s}, \ldots, \rho_{m}^{s}\right)=\mathbb{Q}\left(\tau_{1}^{s}, \ldots, \tau_{n}^{s}\right)$, where $\mathbb{Q}\left(a_{1}, \ldots, a_{m}\right)$ denotes the subfield of $\mathbb{R}$ generated by $\mathbb{Q}$ and $a_{1}, \ldots, a_{m}$.

(2) There exist positive integers $p, q$ such that

$$
\begin{aligned}
& \operatorname{sgp}\left(\rho_{1}^{p}, \ldots, \rho_{m}^{p}\right) \subseteq \operatorname{sgp}\left(\tau_{1}, \ldots, \tau_{n}\right), \\
& \operatorname{sgp}\left(\tau_{1}^{q}, \ldots, \tau_{n}^{q}\right) \subseteq \operatorname{sgp}\left(\rho_{1}, \ldots, \rho_{m}\right),
\end{aligned}
$$

where $\operatorname{sgp}\left(a_{1}, \ldots, a_{m}\right)$ denotes the subsemigroup of $\left(\mathbb{R}^{+}, \times\right)$generated by $a_{1}, \ldots, a_{m}$.

\footnotetext{
${ }^{1}$ One referee told us that Jang-Mei Wu at the University of Illinois at Urbana-Champaign also solved the $\{1,3,4\}-\{1,4,5\}$ problem years ago without publishing.
} 
Using this theorem, it was shown in 6 , that there exist dust-like self-similar sets $E$ and $F$ such that $\operatorname{dim}_{H} E=\operatorname{dim}_{H} F$ but $E$ and $F$ are not Lipschitz equivalent. Also, from this theorem, the following question arises naturally:

Question 1. Can we present non-trivial sufficient conditions and necessary conditions on $\boldsymbol{\rho}$ and $\boldsymbol{\tau}$ such that $\mathcal{D}(\boldsymbol{\rho}) \sim \mathcal{D}(\boldsymbol{\tau})$ ?

Since the above work by Falconer and Marsh, there has been little progress in this direction that we know of. The present paper does not give a complete answer to Question 1, which is likely to be extremely hard. It does, however, answer the question in several important special cases that should allow us to gain some deep insight into the problem.

In 6] Falconer and Marsh had developed several techniques to study the Lipschitz equivalence of dust-like self-similar sets. These techniques allowed them to prove Theorem 1.2 and other important results (see also Lemmas 2.1 and 2.3 and Remark 2.5). Recently some other techniques have been developed. One that will play a significant role in this paper is a result of $\mathrm{Xi}$ and Ruan [18, which states that if $f: E \rightarrow F$ is a bi-Lipschitz map between two dust-like self-similar sets, then $f$ has a certain measure-preserving property. Precisely, there is a cylinder $E_{\mathbf{i}_{0}} \subset E$ such that the restriction of $f$ on $E_{\mathbf{i}_{0}}$ preserves the Hausdorff measure $\mathcal{H}^{s}$ up to a constant (Lemma 2.4). This result generalized the measure-preserving property obtained by Cooper and Pignataro [1] for an order-preserving bi-Lipschitz function between two dust-like subsets of $\mathbb{R}$.

Other conditions on Lipschitz equivalence of self-similar sets have been established, e.g. in $\mathrm{Xi}$ and Ruan [18] and in $\mathrm{Xi}$ [15]. In both studies, sufficient and necessary conditions for Lipschitz equivalence have been established in terms of graph-directed sets. However, these conditions are difficult to check. Generally, given two contraction vectors $\boldsymbol{\rho}=\left(\rho_{1}, \rho_{2}, \ldots, \rho_{m}\right)$ and $\boldsymbol{\tau}=\left(\tau_{1}, \tau_{2}, \ldots, \tau_{n}\right)$, it is not practical to apply these conditions to decide whether $\mathcal{D}(\boldsymbol{\rho})$ and $\mathcal{D}(\boldsymbol{\tau})$ are Lipschitz equivalent, even for the two-branch case $m=n=2$.

In this paper we introduce the notion of rank for a contraction vector $\boldsymbol{\rho}=$ $\left(\rho_{1}, \ldots, \rho_{m}\right)$. Let $\left\langle\rho_{1}, \ldots, \rho_{m}\right\rangle$ denote the subgroup of $\left(\mathbb{R}^{+}, \times\right)$generated by $\rho_{1}, \ldots$, $\rho_{m}$; then it is a free abelian group. It follows that $\left\langle\rho_{1}, \ldots, \rho_{m}\right\rangle$ has a non-empty basis, and we can define the rank of $\left\langle\rho_{1}, \ldots, \rho_{m}\right\rangle$, which we denote by $\operatorname{rank}\langle\boldsymbol{\rho}\rangle$, to be the cardinality of the basis. Clearly $1 \leq \operatorname{rank}\langle\boldsymbol{\rho}\rangle \leq m$. In the case that $\operatorname{rank}\langle\boldsymbol{\rho}\rangle=m$, we say $\boldsymbol{\rho}$ has full rank. For rank of a free abelian group, see e.g. 7 .

According to Theorem $1.2(2)$, if $\mathcal{D}(\boldsymbol{\rho}) \sim \mathcal{D}(\boldsymbol{\tau})$, then $\operatorname{rank}\langle\boldsymbol{\rho}\rangle=\operatorname{rank}\langle\boldsymbol{\tau}\rangle=$ $\operatorname{rank}\langle\boldsymbol{\rho}, \boldsymbol{\tau}\rangle$, where $\langle\boldsymbol{\rho}, \boldsymbol{\tau}\rangle:=\left\langle\rho_{1}, \ldots, \rho_{m}, \tau_{1}, \ldots, \tau_{n}\right\rangle$ for $\boldsymbol{\rho}=\left(\rho_{1}, \ldots, \rho_{m}\right)$ and $\boldsymbol{\tau}=$ $\left(\tau_{1}, \ldots, \tau_{n}\right)$. One of our main theorems is:

Theorem 1.3. Let $\boldsymbol{\rho}=\left(\rho_{1}, \ldots, \rho_{m}\right)$ and $\boldsymbol{\tau}=\left(\tau_{1}, \ldots, \tau_{m}\right)$ be two contraction vectors such that $\operatorname{rank}\langle\boldsymbol{\rho}\rangle=m$. Then $\mathcal{D}(\boldsymbol{\rho})$ and $\mathcal{D}(\boldsymbol{\tau})$ are Lipschitz equivalent if and only if $\boldsymbol{\tau}$ is a permutation of $\boldsymbol{\rho}$.

Theorem 1.3 and a result on the irreducibility of certain trinomials by Ljunggren [9] allows us to completely characterize the Lipschitz equivalence of dust-like selfsimilar sets with two branches. We prove:

Theorem 1.4. Let $\left(\rho_{1}, \rho_{2}\right)$ and $\left(\tau_{1}, \tau_{2}\right)$ be two contraction vectors with $\rho_{1} \leq \rho_{2}$, $\tau_{1} \leq \tau_{2}$. Assume that $\rho_{1} \leq \tau_{1}$. Then $\mathcal{D}(\boldsymbol{\rho}) \sim \mathcal{D}(\boldsymbol{\tau})$ if and only if one of the two 
conditions holds:

(1) $\rho_{1}=\tau_{1}$ and $\rho_{2}=\tau_{2}$.

(2) There exists a real number $0<\lambda<1$ such that

$$
\left(\rho_{1}, \rho_{2}\right)=\left(\lambda^{5}, \lambda\right) \text { and }\left(\tau_{1}, \tau_{2}\right)=\left(\lambda^{3}, \lambda^{2}\right) .
$$

Another case where the Lipschitz equivalence of dust-like self-similar sets can be characterized completely occurs when one of them has a uniform contraction ratio.

Theorem 1.5. Let $\boldsymbol{\rho}=\left(\rho_{1}, \cdots, \rho_{m}\right)=(\rho, \ldots, \rho)$ and $\boldsymbol{\tau}=\left(\tau_{1}, \ldots, \tau_{n}\right)$. Then $\mathcal{D}(\boldsymbol{\rho})$ and $\mathcal{D}(\boldsymbol{\tau})$ are Lipschitz equivalent if and only if the following conditions hold:

(1) $\operatorname{dim}_{H} \mathcal{D}(\boldsymbol{\tau})=\operatorname{dim}_{H} \mathcal{D}(\boldsymbol{\rho})=\log m / \log \rho^{-1}$.

(2) There exists a $q \in \mathbb{Z}^{+}$such that $m^{1 / q} \in \mathbb{Z}$ and

$$
\frac{\log \tau_{j}}{\log \rho} \in \frac{1}{q} \mathbb{Z} \quad \text { for all } \quad j=1,2, \ldots, n .
$$

As an application of Theorem 1.4 we can see that the conditions in Theorem 1.2 are necessary but not sufficient via the following example.

Example 1.1. Let $x, y, 0<x, y<1$, be the solution of the equations

$$
x^{6}+y=1 \text { and } x^{3}+y^{4}=1 .
$$

One can easily check that the solution indeed exists. Let $s$ be a real number such that $0<s<1$. Suppose that the contraction vectors of $E$ and $F$ are $\left(x^{6 / s}, y^{1 / s}\right)$ and $\left(x^{3 / s}, y^{4 / s}\right)$, respectively. Then $E$ and $F$ have the same Hausdorff dimension and satisfy the conditions in Theorem 1.2. However, $E$ and $F$ are not Lipschitz equivalent by Theorem 1.4

To prove Theorem 1.3 in this paper we shall introduce a new equivalent relation between two dust-like self-similar sets, which is referred to as the matchable condition. The matchable condition is somewhat technical, so we shall defer its definition to the next section. We prove a refinement of condition (2) in Theorem 1.2 involving the matchable condition:

Theorem 1.6. Let $E$ and $F$ be two dust-like self-similar sets. If $E \sim F$, then $E$ and $F$ are matchable.

The paper is organized as follows: In Section 2, we review some important results in [6, 18, concerning the Lipschitz equivalence of dust-like self-similar sets, and prove Theorem 1.6. In Section 3, we prove Theorem 1.3. In Section 4, we focus on two-branch self-similar sets and prove Theorem 1.4. Finally in Section 5 we prove Theorem 1.5

\section{A NEW CRITERION FOR LIPSCHITZ EQUIVALENCE}

2.1. Measure-preserving property. We first introduce some notation. Let $E$ be the attractor of the IFS $\Phi=\left\{\phi_{1}, \ldots, \phi_{m}\right\}$. Let $\Sigma_{m}^{*}:=\bigcup_{k=1}^{\infty}\{1,2, \ldots, m\}^{k}$. For any word $\mathbf{i}=i_{1} \cdots i_{k} \in \Sigma_{m}^{*}$, we call $k$ the length of the word $\mathbf{i}$ and denote it by $|\mathbf{i}|$. Furthermore, a cylinder $E_{\mathbf{i}}$ is defined to be $E_{\mathbf{i}}=\phi_{\mathbf{i}}(E):=\phi_{i_{1}} \circ \cdots \circ \phi_{i_{k}}(E)$.

In this section we consider the Lipschitz equivalence of two dust-like self-similar sets $E$ and $F$ with the following setup: We assume that $E$ is the attractor of 
$\Phi=\left\{\phi_{1}, \ldots, \phi_{m}\right\}$ with contraction vector $\boldsymbol{\rho}=\left(\rho_{1}, \ldots, \rho_{m}\right)$ and $F$ is the attractor of $\Psi=\left\{\psi_{1}, \ldots, \psi_{n}\right\}$ with contraction vector $\boldsymbol{\tau}=\left(\tau_{1}, \ldots, \tau_{n}\right)$. We also assume in subsections 2.1 and 2.2 that $s=\operatorname{dim}_{H} E=\operatorname{dim}_{H} F$ and $f: E \longrightarrow F$ is a bi-Lipschitz map.

For any word $\mathbf{i}=i_{1} \cdots i_{k} \in \Sigma_{n}^{*}$, we define $\boldsymbol{\tau}_{\mathbf{i}}=\prod_{j=1}^{k} \tau_{i_{j}}$. Similarly, we can define $\boldsymbol{\rho}_{j}$ for $\mathbf{j} \in \Sigma_{m}^{*}$. The following lemma is fundamental.

Lemma $2.1([6])$. There exists an integer $n_{0}$ such that for any $\mathbf{i} \in \Sigma_{m}^{*}$, there exist $\mathbf{k}, \mathbf{j}_{1}, \ldots, \mathbf{j}_{p} \in \Sigma_{n}^{*}$ such that $F_{\mathbf{k}_{1}}, \ldots, F_{\mathbf{k j}_{p}}$ are disjoint and

$$
f\left(E_{\mathbf{i}}\right)=\bigcup_{r=1}^{p} F_{\mathbf{k j}_{r}} \subset F_{\mathbf{k}},
$$

where each $\left|\mathbf{j}_{r}\right| \leq n_{0}$. In particular, $\mathcal{H}^{s}\left(f\left(E_{\mathbf{i}}\right)\right)=\mathcal{H}^{s}\left(F_{\mathbf{k}}\right) \sum_{r=1}^{p}\left(\boldsymbol{\tau}_{\mathbf{j}_{r}}\right)^{s}$.

Remark 2.2. It is clear that we can require that each $\left|\mathbf{j}_{r}\right|=n_{0}$ in the above lemma. Also, under this restriction, $\mathbf{k}$ is unique if we require $\mathbf{k}$ to have the maximal length. Consequently the set $\left\{\mathbf{j}_{1}, \ldots, \mathbf{j}_{p}\right\}$ is also uniquely determined by $\mathbf{i}$. We will write $p_{\mathbf{i}}$ for $p$ if necessary. We call this unique decomposition the maximum decomposition of $f\left(E_{\mathbf{i}}\right)$ with respect to $F$ and $n_{0}$. From now on, we fix $n_{0}$ in this section. We remark that $p$ in (2.1) is bounded since $p \leq n^{n_{0}}$.

In [6, Falconer and Marsh introduced a function $g_{k}: E \longrightarrow \mathbb{R}$ defined by

$$
g_{k}(x)=\frac{\mathcal{H}^{s}\left(f\left(E_{\mathbf{i}}\right)\right)}{\mathcal{H}^{s}\left(E_{\mathbf{i}}\right)}
$$

for $x \in E_{\mathbf{i}}$, where $\mathbf{i} \in\{1, \ldots, m\}^{k}$. We shall abuse the notation by writing $g_{k}\left(E_{\mathbf{i}}\right)=$ $\frac{\mathcal{H}^{s}\left(f\left(E_{\mathbf{i}}\right)\right)}{\mathcal{H}^{s}\left(E_{\mathbf{i}}\right)}$. It is easy to show that

$$
g_{k}\left(E_{\mathbf{i}}\right)=\sum_{i=1}^{m} \frac{\mathcal{H}^{s}\left(E_{\mathbf{i} i}\right)}{\mathcal{H}^{s}\left(E_{\mathbf{i}}\right)} g_{k+1}\left(E_{\mathbf{i} i}\right) .
$$

Lemma $2.3([6])$. The set $\left\{\frac{g_{k+1}(x)}{g_{k}(x)}: x \in E, k \geq 1\right\}$ is finite.

$\mathrm{Xi}$ and Ruan obtained the following property. We include a short proof for completeness.

Lemma 2.4 ([18]). There is a cylinder $E_{\mathbf{i}_{0}}$ and a constant $c>0$ such that $g_{k}(x)=c$ for all $x \in E_{\mathbf{i}_{0}}$ and $k \geq\left|\mathbf{i}_{0}\right|$.

Proof. Set $T=\sup _{k \geq 1} \max _{|\mathbf{i}|=k} g_{k}\left(E_{\mathbf{i}}\right)$. Since $f$ is bi-Lipschitz, we have $T<+\infty$.

If $\frac{g_{k+1}(x)}{g_{k}(x)}=1$ for all $x \in E$ and all $k \geq 1$, then the lemma clearly holds. Otherwise set $\delta=\min \left(\left\{\left|\frac{g_{k+1}(x)}{g_{k}(x)}-1\right|: x \in E, k \geq 1\right\} \backslash\{0\}\right)$. Then $\delta>0$ by Lemma 2.3. Choose $\mathbf{i}_{0}$ such that (denote $\ell=\left|\mathbf{i}_{0}\right|$ )

$$
g_{\ell}\left(E_{\mathbf{i}_{0}}\right)>T /(1+\delta) \text {. }
$$

Then $\frac{g_{\ell+1}\left(E_{\mathbf{i}_{0} j}\right)}{g_{\ell}\left(E_{\mathbf{i}_{0}}\right)}<1+\delta$ for all $j$, and hence $\frac{g_{\ell+1}\left(E_{\mathbf{i}_{0} j}\right)}{g_{\ell}\left(E_{\mathbf{i}_{0}}\right)} \leq 1$ by the definition of $\delta$.

Now formula (2.3) implies that $\frac{g_{\ell+1}\left(E_{\mathbf{i}_{0} j}\right)}{g_{\ell}\left(E_{\mathbf{i}_{0}}\right)}=1$ for all $j$. Hence each $E_{\mathbf{i}_{0} j}$ satisfies (2.4), and we can repeat the same argument with $E_{\mathbf{i}_{0} j}$ in place of $E_{\mathbf{i}_{0}}$. Set $c=$ $g_{\ell}\left(E_{\mathbf{i}_{0}}\right)$, and the lemma is proved. 
This lemma means that the restriction of $f$ on $E_{\mathbf{i}_{0}}$ is measure-preserving up to a constant. More precisely, for any Borel set $A \subset E_{\mathbf{i}_{0}}$ we have

$$
\frac{\mathcal{H}^{s}(f(A))}{\mathcal{H}^{s}(A)}=c=\frac{\mathcal{H}^{s}\left(f\left(E_{\mathbf{i}_{0}}\right)\right)}{\mathcal{H}^{s}\left(E_{\mathbf{i}_{0}}\right)} \text {. }
$$

Remark 2.5. To prove Theorem 1.2, one needs the fact that $g_{k}$ converges on a set with positive Hausdorff measure $\mathcal{H}^{s}$. [6] showed that $g_{k}(x)$ converges for $\mathcal{H}^{s}$ almost all $x \in E$ by using the martingale convergence theorem. Lemma 2.4 says that $g_{k}(x)$ converges on a cylinder of $E$ and hence provides an alternative proof of Theorem 1.2 .

We shall call the cylinder $E_{\mathbf{i}_{0}}$ in Lemma 2.4 a stable cylinder with respect to the map $f$. From now on, we fix a stable cylinder $E_{\mathbf{i}_{0}}$ in this section. Going back to Lemma 2.1 and Remark 2.2. for any $\mathbf{i} \in \Sigma_{m}^{*}$, there is a (unique) maximum decomposition of $f\left(E_{\mathbf{i}_{0} \mathbf{i}}\right)$ with respect to $F$ and $n_{0}$ :

$$
f\left(E_{\mathbf{i}_{0} \mathbf{i}}\right)=\bigcup_{r=1}^{p_{\mathbf{i}_{0} \mathbf{i}}} F_{\mathbf{k}_{\mathbf{j}_{r}}},
$$

where $\left|\mathbf{j}_{r}\right|=n_{0}$. The following observation is crucial for the proof of our new criterion.

Lemma 2.6. The set $\mathcal{M}=\bigcup_{\mathbf{i} \in \Sigma_{m}^{*}}\left\{\frac{\mathcal{H}^{s}\left(E_{\mathbf{i}_{0} \mathbf{i}}\right)}{\mathcal{H}^{s}\left(F_{\mathbf{k}_{r}}\right)}: 1 \leq r \leq p_{\mathbf{i}_{0} \mathbf{i}}\right\}$ is finite. Consequently, the sets

$$
\mathcal{M}^{\prime}=\bigcup_{\mathbf{i} \in \Sigma_{m}^{*}}\left\{\frac{\operatorname{diam} E_{\mathbf{i}_{0} \mathbf{i}}}{\operatorname{diam} F_{\mathbf{k}_{r}}}: 1 \leq r \leq p_{\mathbf{i}_{0} \mathbf{i}}\right\} \text { and } \mathcal{M}^{\prime \prime}=\bigcup_{\mathbf{i} \in \Sigma_{m}^{*}}\left\{\frac{\rho_{\mathbf{i}_{0} \mathbf{i}}}{\tau_{\mathbf{k j}_{r}}}: 1 \leq r \leq p_{\mathbf{i}_{0} \mathbf{i}}\right\}
$$

are finite.

Proof. Note that

$$
\frac{\mathcal{H}^{s}\left(E_{\mathbf{i}_{\mathbf{i}} \mathbf{i}}\right)}{\mathcal{H}^{s}\left(F_{\mathbf{k}_{r}}\right)}=\frac{\mathcal{H}^{s}\left(E_{\mathbf{i}_{0} \mathbf{i}}\right)}{\sum_{j=1}^{p_{\mathbf{i}_{0} \mathbf{i}}} \mathcal{H}^{s}\left(F_{\mathbf{k j}_{j}}\right)} \cdot \frac{\sum_{j=1}^{p_{\mathbf{i}_{0} \mathbf{i}}} \mathcal{H}^{s}\left(F_{\mathbf{k}_{j}}\right)}{\mathcal{H}^{s}\left(F_{\mathbf{k j}_{r}}\right)}=\frac{1}{c} \frac{\sum_{j=1}^{p_{\mathbf{i}_{0} \mathbf{i}}} \mathcal{H}^{s}\left(F_{\mathbf{k}_{j}}\right)}{\mathcal{H}^{s}\left(F_{\mathbf{k}_{r}}\right)}=\frac{1}{c} \frac{\sum_{j=1}^{p_{\mathbf{i}_{0} \mathbf{i}}} \boldsymbol{\tau}_{\mathbf{j}_{j}}^{s}}{\boldsymbol{\tau}_{\mathbf{j}_{r}}^{s}} .
$$

The last expression can take only finite many values, since $p_{\mathbf{i}_{0} \mathbf{i}} \leq n^{n_{0}}$ and each $\mathbf{j}_{j}$ can take on only finitely many distinct values. It follows that $\mathcal{M}$ is a finite set.

Since $\frac{\mathcal{H}^{s}\left(E_{\mathbf{i}_{\mathbf{i}}}\right)}{\mathcal{H}^{s}\left(F_{\mathbf{k}_{r}}\right)}=c_{0} \cdot\left(\frac{\operatorname{diam} E_{\mathbf{i}_{\mathbf{i}} \mathbf{i}}}{\operatorname{diam} F_{\mathbf{k}_{r}}}\right)^{s}$, where $c_{0}=\frac{\mathcal{H}^{s}(E)}{\mathcal{H}^{s}(F)} \cdot\left(\frac{\operatorname{diam} F}{\operatorname{diam} E}\right)^{s}$ is a constant only dependent on $E$ and $F$, we know that $\mathcal{M}^{\prime}$ is a finite set. It follows from $\frac{\rho_{\mathbf{i}_{0} \mathbf{i}}}{\tau_{\mathbf{k}_{r}}}=\frac{\operatorname{diam} E_{\mathbf{i}_{0} \mathbf{i}}}{\operatorname{diam} F_{\mathbf{k}_{r}}} \cdot \frac{\operatorname{diam} F}{\operatorname{diam} E}$ that $\mathcal{M}^{\prime \prime}$ is also a finite set.

2.2. New criterion. Let $\boldsymbol{\rho}$ and $\boldsymbol{\tau}$ be the contraction vectors in the above subsection. We call $w_{1}, \ldots, w_{L}$ a pseudo-basis of $V=\langle\boldsymbol{\rho}, \boldsymbol{\tau}\rangle$ if $L=\operatorname{rank} V$ and $\left\langle w_{1}, \ldots, w_{L}\right\rangle \supseteq V$. It is clear that a basis of $V$ is natural to be a pseudo-basis. For any $x_{1}, x_{2} \in V$, we define their distance with respect to the pseudo-basis $w_{1}, \ldots, w_{L}$ by

$$
h\left(x_{1}, x_{2}\right):=\left(\sum_{j=1}^{L}\left(s_{j}-t_{j}\right)^{2}\right)^{1 / 2},
$$

where $s_{j}, t_{j} \in \mathbb{Z}$ are the unique integers such that $x_{1}=\prod_{j=1}^{L} w_{j}^{s_{j}}, x_{2}=\prod_{j=1}^{L} w_{j}^{t_{j}}$. It is easy to show that if $h_{1}$ and $h_{2}$ are two distances on $V$ defined as above, then 
they are comparable, i.e., there exists a constant $C \geq 1$ such that

$$
C^{-1} h_{1}\left(x_{1}, x_{2}\right) \leq h_{2}\left(x_{1}, x_{2}\right) \leq C h_{1}\left(x_{1}, x_{2}\right), \quad \forall x_{1}, x_{2} \in V .
$$

Hence, from now on we fix the pseudo-basis and the function $h$. let

Denote $\boldsymbol{\rho}_{\max }=\max \left\{\rho_{1}, \ldots, \rho_{m}\right\}$ and $\boldsymbol{\rho}_{\min }=\min \left\{\rho_{1}, \ldots, \rho_{m}\right\}$. For any $t \in(0,1)$

$$
\mathcal{W}(E, t):=\left\{\mathbf{i} \in \Sigma_{m}^{*}: \boldsymbol{\rho}_{\mathbf{i}} \leq t<\boldsymbol{\rho}_{\mathbf{i}^{*}}\right\},
$$

where $\mathbf{i}^{*}$ is the word obtained by deleting the last letter of $\mathbf{i}$, i.e., $\mathbf{i}^{*}=i_{1} \cdots i_{k-1}$ if $\mathbf{i}=i_{1} \cdots i_{k}$. We define $\boldsymbol{\rho}_{\mathbf{i}^{*}}=1$ if the length of $\mathbf{i}$ equals 1 . Similarly, we may define $\mathcal{W}(F, t)$ with respect to its contraction vector $\tau$. We remark that $\mathcal{W}(E, t)$ has been used in other studies on self-similar sets (e.g. 8, 13]).

Pick some $\mathbf{i} \in \Sigma_{m}^{*}$. There is a (unique) maximum decomposition of $f\left(E_{\mathbf{i}}\right)$ with respect to $F$ and $n_{0}$ :

$$
f\left(E_{\mathbf{i}}\right)=\bigcup_{r=1}^{p_{\mathbf{i}}} F_{\mathbf{k j}_{r}},
$$

where $\left|\mathbf{j}_{r}\right|=n_{0}$. We define a relation $\mathcal{R}(\mathbf{i}, t, f) \subset \mathcal{W}(E, t) \times \mathcal{W}(F, t)$ by

$$
\mathcal{R}(\mathbf{i}, t, f):=\left\{\left(\mathbf{i}^{\prime}, \mathbf{j}^{\prime}\right) \in \mathcal{W}(E, t) \times \mathcal{W}(F, t): f\left(E_{\mathbf{i i}^{\prime}}\right) \cap \bigcup_{r=1}^{p_{\mathbf{i}}} F_{\mathbf{k j}_{r} \mathbf{j}^{\prime}} \neq \emptyset\right\} .
$$

We need the following geometrical lemma to prove our criterion. Note that $F$ is dust-like and satisfies the open set condition, i.e., there exists an open set $V$, such that $V \supset \bigcup_{i=1}^{n} \psi_{i}(V)$ and $\psi_{i}(V) \cap \psi_{j}(V)=\emptyset$ for distinct $i, j$. Thus, using the method in [13], we can easily see that the following lemma holds (for a detailed proof, please see Appendix A).

Lemma 2.7. For any two positive numbers $c_{1}, c_{2}$ with $c_{1} \leq c_{2}$, there exists a constant $c_{3}>0$ such that for any non-empty subset $A$ of $\mathbb{R}^{d}, A$ can intersect at most $c_{3}$ mutually disjoint cylinders $F_{\mathbf{i}}$ with $c_{1} \operatorname{diam} A \leq \operatorname{diam} F_{\mathbf{i}} \leq c_{2} \operatorname{diam} A$.

Now we can prove our criterion.

Theorem 2.8. Assume that $f: E \longrightarrow F$ is bi-Lipschitz and $\mathbf{i}_{0} \in \Sigma_{m}^{*}$ is a stable cylinder. Let $h$ be a distance on $V=\langle\boldsymbol{\rho}, \boldsymbol{\tau}\rangle$ defined by (2.6). Then there exists a constant $M_{0}>0$ such that for any $t \in(0,1)$ we have:

(1) For any $\mathbf{i} \in \mathcal{W}(E, t)$,

$$
1 \leq \operatorname{card}\left\{\mathbf{j}:(\mathbf{i}, \mathbf{j}) \in \mathcal{R}\left(\mathbf{i}_{0}, t, f\right)\right\} \leq M_{0}
$$

Similarly, for any $\mathbf{j} \in \mathcal{W}(F, t), 1 \leq \operatorname{card}\left\{\mathbf{i}:(\mathbf{i}, \mathbf{j}) \in \mathcal{R}\left(\mathbf{i}_{0}, t, f\right)\right\} \leq M_{0}$.

(2) If $(\mathbf{i}, \mathbf{j}) \in \mathcal{R}\left(\mathbf{i}_{0}, t, f\right)$, then $h\left(\boldsymbol{\rho}_{\mathbf{i}}, \boldsymbol{\tau}_{\mathbf{j}}\right) \leq M_{0}$.

Proof. Let $f\left(E_{\mathbf{i}_{0}}\right)=\bigcup_{r=1}^{p} F_{\mathbf{k j}_{r}}$ be the (unique) maximum decomposition of $f\left(E_{\mathbf{i}_{0}}\right)$ with respect to $F$ and $n_{0}$, where $\left|\mathbf{j}_{r}\right|=n_{0}$ and $p=p_{\mathbf{i}_{0}}$.

Fix $t \in(0,1)$. Then

$$
\mathcal{E}=\left\{E_{\mathbf{i}_{0} \mathbf{i}}: \mathbf{i} \in \mathcal{W}(E, t)\right\} \text { and } \mathcal{F}=\left\{F_{\mathbf{k j}_{r} \mathbf{j}}: 1 \leq r \leq p, \mathbf{j} \in \mathcal{W}(F, t)\right\}
$$

is a partition of $E_{\mathbf{i}_{0}}$ and $f\left(E_{\mathbf{i}_{0}}\right)$, respectively, since

$$
\bigcup_{\mathbf{i} \in \mathcal{W}(E, t)} f\left(E_{\mathbf{i}_{0} \mathbf{i}}\right)=f\left(E_{\mathbf{i}_{0}}\right)=\bigcup_{r=1}^{p} F_{\mathbf{k j}_{r}}=\bigcup_{\mathbf{j} \in \mathcal{W}(F, t)} \bigcup_{r=1}^{p} F_{\mathbf{k j}_{r} \mathbf{j}}
$$


By symmetry, in order to prove (1) it suffices to prove (2.8). The left hand side inequality is obvious since for any $E_{\mathbf{i}_{0} \mathbf{i}} \in \mathcal{E}, f\left(E_{\mathbf{i}_{0} \mathbf{i}}\right)$ intersects at least one element of $\mathcal{F}$.

To prove the right hand side inequality of (2.8), we first show that the size of $E_{\mathbf{i}_{0} \mathbf{i}}$ and $F_{\mathbf{k j}_{r} \mathbf{j}}$ are comparable. Indeed, $\frac{\operatorname{diam} E_{\mathbf{i}_{\mathbf{i}}}}{\operatorname{diam} F_{\mathbf{k}_{\mathbf{j}} \mathbf{j}}}=\frac{\operatorname{diam} E}{\operatorname{diam} F} \cdot \frac{\rho_{\mathbf{i}_{0}} \rho_{\mathbf{i}}}{\boldsymbol{\tau}_{\mathbf{k}} \boldsymbol{j}_{r} \boldsymbol{\tau}_{\mathbf{j}}}$. Since $\left\{\mathbf{i}_{0}, \mathbf{k j}_{1}, \ldots, \mathbf{k j}_{p}\right\}$ is fixed, we know that $\frac{\rho_{\mathbf{i}_{0}}}{\tau_{\mathbf{k j}_{r}}}$ takes values from a finite set. Meanwhile $\boldsymbol{\rho}_{\min } \leq \frac{\rho_{\mathbf{i}}}{\tau_{\mathrm{j}}} \leq$ $\frac{1}{\tau_{\min }}$ by the definition of $\mathcal{W}(E, t)$ and $\mathcal{W}(F, t)$. Thus, there exists a constant $C_{0}>0$ such that

$$
C_{0}^{-1}<\frac{\operatorname{diam} E_{\mathbf{i}_{0} \mathbf{i}}}{\operatorname{diam} F_{\mathbf{k j}_{r} \mathbf{j}}}<C_{0}
$$

Combining (2.9) with the bi-Lipschitz property of $f$, we know that there exists a constant $C_{1}>0$ such that $C_{1}^{-1}<\frac{\operatorname{diam} f\left(E_{\mathbf{i}_{0} \mathbf{i}}\right)}{\operatorname{diam} F_{\mathbf{k}_{r} \mathbf{j}}}<C_{1}$. By Lemma 2.7 the number of such $F_{\mathbf{k}_{\mathbf{j}} \mathbf{j}}$ which intersects $f\left(E_{\mathbf{i}_{0} \mathbf{i}}\right)$ is bounded by a constant $M_{0}$ dependent on $C_{1}$, the dimension $d$ of the space and the IFS $\left\{\psi_{i}\right\}_{i=1}^{n}$. In other words,

$$
\max _{\mathbf{i} \in \mathcal{W}(E, t)} \operatorname{card}\left\{\mathbf{j}: \quad(\mathbf{i}, \mathbf{j}) \in \mathcal{R}\left(\mathbf{i}_{0}, t, f\right)\right\}<M_{0} .
$$

We now complete the proof by proving (2). Suppose $(\mathbf{i}, \mathbf{j}) \in \mathcal{R}\left(\mathbf{i}_{0}, t, f\right)$. Then by definition there exists an $r \in\{1, \ldots, p\}$ such that $f\left(E_{\mathbf{i}_{0} \mathbf{i}}\right) \cap F_{\mathbf{k}_{r} \mathbf{j}} \neq \emptyset$. Let us fix this $F_{\mathbf{k}_{\mathbf{j}} \mathbf{j}}$ for the discussions below.

Let $f\left(E_{\mathbf{i}_{0} \mathbf{i}}\right)=\bigcup_{t=1}^{p_{\mathbf{i}_{0} \mathbf{i}}} F_{\mathbf{k}^{\prime} \mathbf{j}_{t}^{\prime}}$ be the maximum decomposition of $f\left(E_{\mathbf{i}_{0} \mathbf{i}}\right)$ with respect to $F$ and $n_{0}$, where $\left|\mathbf{j}_{t}^{\prime}\right|=n_{0}$. Then there is a $t$ such that $F_{\mathbf{k}^{\prime} \mathbf{j}_{t}^{\prime}} \cap F_{\mathbf{k j}_{r} \mathbf{j}} \neq \emptyset$. Since $F_{\mathbf{k}^{\prime} \mathbf{j}_{t}^{\prime}}$ and $F_{\mathbf{k j}_{r} \mathbf{j}}$ are all cylinders, we have

$$
F_{\mathbf{k}^{\prime} \mathbf{j}_{t}^{\prime}} \subset F_{\mathbf{k}_{r} \mathbf{j}} \quad \text { or } \quad F_{\mathbf{k j}_{r} \mathbf{j}} \subset F_{\mathbf{k}^{\prime} \mathbf{j}_{t}^{\prime}}
$$

Notice that

$$
\frac{\rho_{\mathbf{i}}}{\tau_{\mathbf{j}}}=\frac{\rho_{\mathbf{i}_{0} \mathbf{i}}}{\tau_{\mathbf{k j}_{r} \mathbf{j}}} \cdot \frac{\tau_{\mathbf{k j}_{r}}}{\rho_{\mathbf{i}_{0}}}=\frac{\rho_{\mathbf{i}_{0} \mathbf{i}}}{\tau_{\mathbf{k}^{\prime} \mathbf{j}_{t}^{\prime}}} \cdot \frac{\tau_{\mathbf{k}^{\prime} \mathbf{j}_{t}^{\prime}}}{\tau_{\mathbf{k j}_{r} \mathbf{j}}} \cdot \frac{\tau_{\mathbf{k j}_{r}}}{\rho_{\mathbf{i}_{0}}} .
$$

By Lemma 2.6, we know that $\frac{\rho_{\mathbf{i}_{0} \mathbf{i}}}{\tau_{\mathbf{k}^{\prime} \mathbf{j}_{t}^{\prime}}}$ takes values from a finite set $\mathcal{M}^{\prime \prime}$. On the other hand, $\frac{\tau_{\mathbf{k} \mathbf{j}_{r}}}{\rho_{\mathbf{i}_{0}}}$ takes only finitely many values since $\left\{\mathbf{i}_{0}, \mathbf{k j}_{1}, \ldots, \mathbf{k} \mathbf{j}_{p}\right\}$ is fixed. Thus, in order to prove (2), it suffices to prove that $\frac{\boldsymbol{\tau}_{\mathbf{k}^{\prime} \mathbf{j}_{t}^{\prime}}}{\boldsymbol{\tau}_{\mathbf{k}_{r} \mathbf{j}}}$ belongs to a finite set.

By Lemma 2.6 $\frac{\operatorname{diam} E_{\mathbf{i}_{0} \mathbf{i}}}{\operatorname{diam} F_{\mathbf{k}^{\prime} j_{t}^{\prime}}}$ takes values from a finite set $\mathcal{M}^{\prime}$. Combining this with (2.9), we know that $\operatorname{diam} F_{\mathbf{k j}_{r} \mathbf{j}}$ and $\operatorname{diam} F_{\mathbf{k}^{\prime} \mathbf{j}_{t}^{\prime}}$ are comparable. Thus, using (2.10), we obtain that $\frac{\operatorname{diam} F_{\mathbf{k}^{\prime} \mathbf{j}_{t}^{\prime}}}{\operatorname{diam} F_{\mathbf{k}_{\mathbf{j}_{r} \mathbf{j}}}}$ belongs to a finite set so that $\frac{\boldsymbol{\tau}_{\mathbf{k}^{\prime} \mathbf{j}_{t}^{\prime}}}{\boldsymbol{\tau}_{\mathbf{k}_{\mathbf{j}_{r} \mathbf{j}}}}$ belongs to a finite set.

2.3. Matchable condition. Let $E$ and $F$ be two dust-like self-similar sets with contraction vectors $\boldsymbol{\rho}$ and $\boldsymbol{\tau}$, respectively. Let $h$ be a distance on $V=\langle\boldsymbol{\rho}, \boldsymbol{\tau}\rangle$ defined by (2.6) .

Let $M_{0}$ be a constant. For $t \in(0,1)$, a relation $\mathcal{R} \subset \mathcal{W}(E, t) \times \mathcal{W}(F, t)$ is said to be $\left(M_{0}, h\right)$-matchable, or simply $M_{0}$-matchable if there is no confusion, if:

(i) $1 \leq \operatorname{card}\{\mathbf{j}:(\mathbf{i}, \mathbf{j}) \in \mathcal{R}\} \leq M_{0}$ for any $\mathbf{i} \in \mathcal{W}(E, t)$ and $1 \leq \operatorname{card}\{\mathbf{i}:(\mathbf{i}, \mathbf{j}) \in$ $\mathcal{R}\} \leq M_{0}$ for any $\mathbf{j} \in \mathcal{W}(F, t)$.

(ii) If $(\mathbf{i}, \mathbf{j}) \in \mathcal{R}$, then $h\left(\boldsymbol{\rho}_{i}, \boldsymbol{\tau}_{j}\right) \leq M_{0}$.

We also say that $\mathcal{W}(E, t)$ and $\mathcal{W}(F, t)$ are $\left(M_{0}, h\right)$-matchable, or $M_{0}$-matchable, if there exists an $\left(M_{0}, h\right)$-matchable relation $\mathcal{R} \subset \mathcal{W}(E, t) \times \mathcal{W}(F, t)$. 
Definition 2.9. We shall call two self-similar sets $E$ and $F$ matchable if there exists a constant $M_{0}$ such that for any $t \in(0,1), \mathcal{W}(E, t)$ and $\mathcal{W}(F, t)$ are $M_{0}$-matchable.

We remark that the matchable property does not depend on the choice of pseudobasis of $\langle\rho, \tau\rangle$.

The proof of Theorem 2.8, which states that if $E \sim F$, then $E$ and $F$ are matchable, yields Theorem 1.6 .

\section{Self-Similar Sets With FUll Algebraic RANK}

For each contraction vector $\boldsymbol{\rho}=\left(\rho_{1}, \ldots, \rho_{m}\right)$ we defined $\operatorname{rank}\langle\boldsymbol{\rho}\rangle$ to be the cardinality of the basis of the multiplication subgroup generated by $\left\{\rho_{j}\right\}$. We shall also define the algebraic rank of any $E \in \mathcal{D}(\boldsymbol{\rho})$ to be $\operatorname{rank}\langle\boldsymbol{\rho}\rangle$. When the algebraic rank is $m$ we say that $E$ and $\mathcal{D}(\boldsymbol{\rho})$ have full algebraic rank. By Theorem 1.2 if two dust-like self-similar sets $E$ and $F$ are Lipschitz equivalent, then they must have the same algebraic rank.

Lemma 3.1. Let $\boldsymbol{\rho}=\left(\rho_{1}, \cdots, \rho_{m}\right)$ and $\boldsymbol{\tau}=\left(\tau_{1}, \ldots, \tau_{m}\right)$ be two contraction vectors such that $\operatorname{rank}\langle\boldsymbol{\rho}\rangle=\operatorname{rank}\langle\boldsymbol{\tau}\rangle=m$. If $\mathcal{D}(\boldsymbol{\rho}) \sim \mathcal{D}(\boldsymbol{\tau})$, then there exist $\lambda_{j} \in \mathbb{R}^{+}, p_{j} \in$ $\mathbb{Z}^{+}, q_{j} \in \mathbb{Z}^{+}, 1 \leq j \leq m$, and a permutation $\kappa$ on $\{1, \ldots, m\}$ such that $\rho_{j}=\lambda_{j}^{p_{j}}$, $\tau_{j}=\lambda_{\kappa(j)}^{q_{\kappa(j)}}, 1 \leq j \leq m$.

Proof. By Theorem 1.2 (2), there exists an integer $p>0$ such that $\tau_{1}, \ldots, \tau_{m}$ belong to the semigroup generated by $\rho_{1}^{1 / p}, \ldots, \rho_{m}{ }^{1 / p}$. Denote $\rho_{j}^{1 / p}$ by $\lambda_{j}$ for each $j$. Then $\lambda_{1}, \ldots, \lambda_{m}$ is a pseudo-basis of $V=\langle\boldsymbol{\rho}, \boldsymbol{\tau}\rangle$. Let $h$ be the distance on $V$ with respect to this pseudo-basis. Let $a_{j i}, 1 \leq i, j \leq m$, be non-negative integers such that $\ln \tau_{j}=a_{j 1} \ln \lambda_{1}+\cdots+a_{j m} \ln \lambda_{m}$. Fix $1 \leq i \leq m$. We assert that there exists at least one $j, 1 \leq j \leq m$, such that $\tau_{j}$ is a power of $\lambda_{i}$, in other words, $\ln \tau_{j}$ is an integral multiple of $\ln \lambda_{i}$.

Without loss of generality, we assume that $i=1$. Suppose $\ln \tau_{j}$ are not integral multiple of $\ln \lambda_{1}$ for all $1 \leq j \leq m$. This means that $\left(a_{j 1}, \ldots, a_{j m}\right)$ does not have the form $(a, 0, \ldots, 0)$.

$E \sim F$ implies that there exists $M_{0}>0$ such that $\mathcal{W}(E, t)$ and $\mathcal{W}(F, t)$ are $\left(M_{0}, h\right)$-matchable for any $t \in(0,1)$. Let $\mathbf{i}=1^{k}=1 \cdots 1$ be an element of $\mathcal{W}(E, t)$. Then there exists $\mathbf{j} \in \mathcal{W}(F, t)$ such that $h\left(\boldsymbol{\rho}_{\mathbf{i}}, \boldsymbol{\tau}_{\mathbf{j}}\right)<M_{0}$. Suppose that the occurrence of the letter $j$ in $\mathbf{j}$ is $c_{j}, 1 \leq j \leq m$. Then

$$
\ln \boldsymbol{\tau}_{\mathbf{j}}=\sum_{i=1}^{m}\left(\sum_{j=1}^{m} c_{j} a_{j i}\right) \ln \lambda_{i} .
$$

Since $\ln \boldsymbol{\rho}_{\mathbf{i}}=k p \ln \lambda_{1}$, we have

$$
h\left(\boldsymbol{\rho}_{\mathbf{i}}, \boldsymbol{\tau}_{\mathbf{j}}\right) \geq \max \left\{\sum_{j=1}^{m} c_{j} a_{j i}: 2 \leq i \leq m\right\} .
$$

Pick any $j \in\{1, \ldots, m\}$. Since $\left(a_{j 1}, \ldots, a_{j m}\right)$ does not have the form $(a, 0, \ldots, 0)$, there exists at least one $i \in\{2, \ldots, m\}$ such that $a_{j i} \geq 1$. Thus $\sum_{j=1}^{m} c_{j} a_{j i} \geq c_{j}$. By the arbitrariness of $j$, we have $M_{0}>h\left(\boldsymbol{\rho}_{\mathbf{i}}, \boldsymbol{\tau}_{\mathbf{j}}\right) \geq \max _{j=1}^{m} c_{j}$. However, $\max c_{j}$ tends to infinity when $t$ tends to 0 . This is a contradiction. Hence our assertion holds. 
Therefore, for any $1 \leq i \leq m$, there exists at least one $j$ such that $\ln \tau_{j}=q_{i} \ln \lambda_{i}$. Moreover, this $j=j(i)$ is unique since $\operatorname{rank}\langle\boldsymbol{\rho}\rangle=\operatorname{rank}\langle\boldsymbol{\tau}\rangle=m$. Let $\kappa$ be the permutation of $1, \ldots, m$ which sends $j$ to $i$; then we have $\ln \tau_{j}=q_{\kappa(j)} \ln \lambda_{\kappa(j)}$.

Setting $p_{j}=p$ for $1 \leq j \leq m$, we obtain the lemma.

Lemma 3.2. Let $m$ be a given positive integer and $G$ be the function defined by

$$
G\left(x_{1}, \ldots, x_{m}\right)=\left(\frac{x_{1}+\cdots+x_{m}}{x_{1}}\right)^{x_{1}} \cdots\left(\frac{x_{1}+\cdots+x_{m}}{x_{m}}\right)^{x_{m}}
$$

where $x_{1}, \ldots, x_{m} \in \mathbb{R}^{+}$. Assume that $a_{1}, \ldots, a_{m}$ are positive real numbers such that

$$
G\left(x_{1}, \ldots, x_{m}\right)=G\left(a_{1} x_{1}, \ldots, a_{m} x_{m}\right)
$$

holds for any positive rational vector $\left(x_{1}, \ldots, x_{m}\right)$. Then $a_{1}=\cdots=a_{m}=1$.

Proof. By the continuity of $G$, we know that (3.2) holds for any positive vectors $\left(x_{1}, \ldots, x_{m}\right)$. For given $x_{1}, x_{2} \in \mathbb{R}^{+}$let $x_{j} \rightarrow 0^{+}$for any $j \geq 3$. It follows from $\lim _{x \rightarrow 0^{+}} x^{x}=1$ and (3.2) that

$$
\left(\frac{x_{1}+x_{2}}{x_{1}}\right)^{x_{1}}\left(\frac{x_{1}+x_{2}}{x_{2}}\right)^{x_{2}}=\left(\frac{a_{1} x_{1}+a_{2} x_{2}}{a_{1} x_{1}}\right)^{a_{1} x_{1}}\left(\frac{a_{1} x_{1}+a_{2} x_{2}}{a_{2} x_{2}}\right)^{a_{2} x_{2}} .
$$

Now we fix $x_{2} \in \mathbb{R}^{+}$and let $x_{1} \rightarrow+\infty$. Then $\left(\frac{x_{1}+x_{2}}{x_{1}}\right)^{x_{1}}$ and $\left(\frac{a_{1} x_{1}+a_{2} x_{2}}{a_{1} x_{1}}\right)^{a_{1} x_{1}}$ converge to $e^{x_{2}}$ and $e^{a_{2} x_{2}}$, respectively. On the other hand, as $x_{1} \rightarrow+\infty$ we have

$$
\left(\frac{x_{1}+x_{2}}{x_{2}}\right)^{x_{2}} \asymp x_{1}^{x_{2}}, \quad\left(\frac{a_{1} x_{1}+a_{2} x_{2}}{a_{2} x_{2}}\right)^{a_{2} x_{2}} \asymp x_{1}^{a_{2} x_{2}},
$$

where $f(x) \asymp g(x)(x \rightarrow+\infty)$ means that there exist constants $c_{1}, c_{2}>0$ such that $c_{1} g(x) \leq f(x) \leq c_{2} g(x)$ for sufficiently large $x$. The equality (3.3) now implies $a_{2}=1$. By symmetry we also have all $a_{j}=1$, proving the lemma.

Lemma 3.3. Let $\boldsymbol{\rho}=\left(\rho_{1}, \ldots, \rho_{m}\right)$ and $\boldsymbol{\tau}=\left(\tau_{1}, \ldots, \tau_{m}\right)$ be two contraction vectors, where for each $j, \rho_{j}=\lambda_{j}^{p_{j}}$ and $\tau_{j}=\lambda_{j}^{q_{j}}$ for some $\lambda_{j}>0$ and $p_{j}, q_{j} \in \mathbb{Z}^{+}$. Assume that $\log \lambda_{1}, \ldots, \log \lambda_{m}$ are linearly independent over $\mathbb{Q}$. Then $\mathcal{D}(\boldsymbol{\rho})$ and $\mathcal{D}(\boldsymbol{\tau})$ are Lipschitz equivalent if and only if $\boldsymbol{\rho}=\boldsymbol{\tau}$.

Proof. Clearly all we need is to prove the only if part. Assume that $\mathcal{D}(\boldsymbol{\rho}) \sim \mathcal{D}(\boldsymbol{\tau})$. Let $E \in \mathcal{D}(\boldsymbol{\rho}), F \in \mathcal{D}(\boldsymbol{\tau})$. Let $h$ be the distance on $V=\langle\boldsymbol{\rho}, \boldsymbol{\tau}\rangle$ with respect to the pseudo-basis $\lambda_{1}, \ldots, \lambda_{m} . E \sim F$ implies that $E$ and $F$ are $\left(M_{0}, h\right)$-matchable for some $M_{0}>0$. Using the matchable property we will prove that $p_{j}=q_{j}$ for $1 \leq j \leq m$.

Given positive integers $A_{1}, \ldots, A_{m}$, set $t=\prod_{j=1}^{m} \lambda_{j}^{p_{j} A_{j}}$ and define $\mathcal{I}=\{\mathbf{i} \in$ $\left.\Sigma_{m}^{*}: \rho_{\mathbf{i}}=t\right\}$. Then $\mathcal{I} \subset \mathcal{W}(E, t)$ and the cardinality of $\mathcal{I}$ is

$$
K\left(A_{1}, \ldots, A_{m}\right):=\operatorname{card} \mathcal{I}=\frac{\left(A_{1}+\cdots+A_{m}\right) !}{A_{1} ! \cdots A_{m} !} .
$$

Let $\mathcal{R}_{t}$ be an $M_{0}$-matchable relation between $\mathcal{W}(E, t)$ and $\mathcal{W}(F, t)$. Let $\mathcal{J}$ be the set of elements $\mathbf{j}$ in $\mathcal{W}(F, t)$ such that $\left\{\mathbf{i} \in \mathcal{I}:(\mathbf{i}, \mathbf{j}) \in \mathcal{R}_{t}\right\} \neq \emptyset$. Then card $\mathcal{J} \geq$ $M_{0}^{-1} \operatorname{card} \mathcal{I}$. Hence

$$
\operatorname{card}\left\{\mathbf{j} \in \mathcal{W}(F, t): h\left(t, \boldsymbol{\tau}_{\mathbf{j}}\right) \leq M_{0}\right\} \geq \operatorname{card} \mathcal{J} \geq M_{0}^{-1} K\left(A_{1}, \ldots, A_{m}\right) .
$$


By the assumption, $\boldsymbol{\tau}_{\mathbf{j}}$ has the form $\boldsymbol{\tau}_{\mathbf{j}}=\prod_{j=1}^{m} \lambda_{j}^{q_{j} B_{j}}$ where $B_{j}$ are non-negative integers. So $\mathbf{j} \in \mathcal{J}$ implies that $h\left(t, \boldsymbol{\tau}_{\mathbf{j}}\right) \leq M_{0}$ and thus $\left|p_{j} A_{j}-q_{j} B_{j}\right| \leq M_{0}$ for $1 \leq j \leq m$. Therefore,

$$
\sum_{\left(B_{1}, \ldots, B_{m}\right)} \frac{\left(B_{1}+\cdots+B_{m}\right) !}{B_{1} ! \cdots B_{m} !} \geq \operatorname{card} \mathcal{J} \geq M_{0}^{-1} K\left(A_{1}, \ldots, A_{m}\right),
$$

where $\left(B_{1}, \ldots, B_{m}\right)$ runs over positive integer vectors satisfying $\left|p_{j} A_{j}-q_{j} B_{j}\right| \leq M_{0}$ for $1 \leq j \leq m$.

Let $C$ be an integer constant such that $\left|B_{j}-\frac{p_{j}}{q_{j}} A_{j}\right|<\frac{M_{0}}{q_{j}}<C, 1 \leq j \leq m$. Set $a_{j}=p_{j} / q_{j}$ for $1 \leq j \leq m$. Then the terms on the left hand side of (3.4) have

$$
\begin{gathered}
\frac{\left(B_{1}+\cdots+B_{m}\right) !}{B_{1} ! \cdots B_{m} !} K^{-1}\left(a_{1} A_{1}, \ldots, a_{m} A_{m}\right) \\
\leq \frac{\left(\frac{p_{1}}{q_{1}} A_{1}+\cdots+\frac{p_{m}}{q_{m}} A_{m}+m C\right) !}{\left(\frac{p_{1}}{q_{1}} A_{1}-C\right) ! \cdots\left(\frac{p_{m}}{q_{m}} A_{m}-C\right) !} \cdot \frac{\left(\frac{p_{1}}{q_{1}} A_{1}\right) ! \cdots\left(\frac{p_{m}}{q_{m}} A_{m}\right) !}{\left(\frac{p_{1}}{q_{1}} A_{1}+\cdots+\frac{p_{m}}{q_{m}} A_{m}\right) !} \\
=\left(\frac{p_{1}}{q_{1}} A_{1}+\cdots+\frac{p_{m}}{q_{m}} A_{m}+m C\right) \cdots\left(\frac{p_{1}}{q_{1}} A_{1}+\cdots+\frac{p_{m}}{q_{m}} A_{m}+1\right) \\
\quad \prod_{j=1}^{m}\left(\frac{p_{j}}{q_{j}} A_{j}\right) \cdots\left(\frac{p_{j}}{q_{j}} A_{j}-C+1\right) .
\end{gathered}
$$

Let $\left(x_{1}, \ldots, x_{m}\right) \in \mathbb{Q}^{m}$ be a positive rational vector. Set $A_{j}=x_{j} q n$, where $q$ is chosen so that all $q x_{j} / q_{j}, q x_{j} / p_{j}$ are integers. Then the left hand side of (3.4) contains at most $(2 C+1)^{m}$ terms, and each term in the sum is not larger than $P(n) K\left(a_{1} A_{1}, \ldots, a_{m} A_{m}\right)$, where $P(n)$ is the polynomial

$$
P(n)=(L n+m C) \cdots(L n+1) \cdot \prod_{j=1}^{m}\left(a_{j} x_{j} q n\right) \cdots\left(a_{j} x_{j} q n-C+1\right),
$$

where $L=\left(a_{1} x_{1}+\cdots+a_{m} x_{m}\right) q$. Hence by (3.4),

$$
(2 C+1)^{m} P(n) K\left(a_{1} x_{1} q n, \ldots, a_{m} x_{m} q n\right) \geq M_{0}^{-1} K\left(x_{1} q n, \ldots, x_{m} q n\right),
$$

and therefore

$$
\frac{K\left(x_{1} q n, \ldots, x_{m} q n\right)}{K\left(a_{1} x_{1} q n, \ldots, a_{m} x_{m} q n\right)} \leq M_{0}(2 C+1)^{m} P(n) .
$$

Similarly, let $C^{\prime}$ be an integer constant such that $\left|A_{j}-\frac{q_{j}}{p_{j}} B_{j}\right|<\frac{M_{0}}{p_{j}}<C^{\prime}, 1 \leq$ $j \leq m$. Set $b_{j}=a_{j}^{-1}=q_{j} / p_{j}, y_{j}=x_{j} a_{j}$ and $B_{j}=y_{j} q n$ for $1 \leq j \leq m$. Then $B_{j}=x_{j} p_{j} q n / q_{j}$ are all integers. Also, $b_{j} y_{j} q n=x_{j} q n=A_{j}$ are all integers. Using Theorem 2.8 and by the same method used for proving (3.5), we obtain

$$
\frac{K\left(y_{1} q n, \ldots, y_{m} q n\right)}{K\left(b_{1} y_{1} q n, \ldots, b_{m} y_{m} q n\right)} \leq M_{0}\left(2 C^{\prime}+1\right)^{m} Q(n)
$$

where $Q(n)$ is a polynomial determined by $p_{j}, q_{j}, x_{j}, q$ and $C^{\prime}$. It follows from (3.5) and (3.6) that

$$
\frac{1}{M_{0}\left(2 C^{\prime}+1\right)^{m} Q(n)} \leq \frac{K\left(x_{1} q n, \ldots, x_{m} q n\right)}{K\left(a_{1} x_{1} q n, \ldots, a_{m} x_{m} q n\right)} \leq M_{0}(2 C+1)^{m} P(n) .
$$


Now Stirling's formula asserts that

$$
n !=\sqrt{2 \pi n}\left(\frac{n}{e}\right)^{n} e^{\frac{\theta(n)}{12 n}}, \quad 0<\theta(n)<1 .
$$

Denote $\theta\left(x_{1} q n+\cdots+x_{m} q n\right), \theta\left(x_{i} q n\right), \theta\left(a_{1} x_{1} q n+\cdots+a_{m} x_{m} q n\right)$ and $\theta\left(a_{i} x_{i} q n\right)$ by $\alpha_{n}, \alpha_{i, n}, \beta_{n}$ and $\beta_{i, n}, 1 \leq i \leq m$, respectively. We have

$$
\begin{aligned}
& \frac{K\left(x_{1} q n, \ldots, x_{m} q n\right)}{K\left(a_{1} x_{1} q n, \ldots, a_{m} x_{m} q n\right)} \\
& \quad=\sqrt{\frac{\left(x_{1}+\cdots+x_{m}\right) a_{1} \cdots a_{m}}{a_{1} x_{1}+\cdots+a_{m} x_{m}}} \cdot e^{\xi_{n}} \cdot\left(\frac{G\left(x_{1}, \ldots, x_{m}\right)}{G\left(a_{1} x_{1}, \ldots, a_{m} x_{m}\right)}\right)^{q n},
\end{aligned}
$$

where $G$ is defined by (3.1) and

$$
\xi_{n}=\frac{1}{12 q n}\left\{\frac{\alpha_{n}}{x_{1}+\cdots+x_{m}}-\sum_{i=1}^{m} \frac{\alpha_{i, n}}{x_{i}}-\frac{\beta_{n}}{a_{1} x_{1}+\cdots+a_{m} x_{m}}+\sum_{i=1}^{m} \frac{\beta_{i, n}}{a_{i} x_{i}}\right\} .
$$

Clearly, for fixed positive rational numbers $a_{i}, x_{i}, 1 \leq i \leq m$, and a fixed positive integer $q$, we have $-1<\xi_{n}<1$ if $n$ is large enough. Thus, there exist two positive constants $c_{1}, c_{2}$ dependent only on $a_{i}, x_{i}, 1 \leq i \leq m$, and $q$ such that

$$
\frac{K\left(x_{1} q n, \ldots, x_{m} q n\right)}{K\left(a_{1} x_{1} q n, \ldots, a_{m} x_{m} q n\right)}=T_{n} \cdot\left(\frac{G\left(x_{1}, \ldots, x_{m}\right)}{G\left(a_{1} x_{1}, \ldots, a_{m} x_{m}\right)}\right)^{q n},
$$

where $0<c_{1}<T_{n}<c_{2}$.

Assume that $\left(a_{1}, \ldots, a_{m}\right) \neq(1, \ldots, 1)$. By Lemma 3.2, we can find a positive rational vector $\left(x_{1}, \ldots, x_{m}\right)$ such that $G\left(x_{1}, \ldots, x_{m}\right) / G\left(a_{1} x_{1}, \ldots, a_{m} x_{m}\right) \neq 1$ and so that (3.8) contradicts (3.7). Thus $p_{j}=q_{j}$ for all $j$ and $\boldsymbol{\rho}=\boldsymbol{\tau}$.

The combination of Lemma 3.1 and Lemma 3.3 immediately yields Theorem 1.3 .

\section{Two-BRAnCh DUst-Like CANTOR SEtS}

In this section we focus on two-branch dust-like self-similar sets, i.e., dust-like self-similar sets generated by two contractions $\mathcal{D}\left(\rho_{1}, \rho_{2}\right)$ and prove Theorem 1.4 We will first need to introduce some results on polynomials with integer coefficients.

Consider the polynomial $f(x)=x^{n}+x^{m}-1$ where $n>m>0$. It is easy to show that there exists a unique $x_{0} \in(0,1)$ such that $f\left(x_{0}\right)=0$. We denote this root $x_{0}$ by $r_{n, m}$.

Proposition 4.1 ([9], Theorem 3). Let $n \geq 2 m>0$. Write $n=n_{1} \ell, m=m_{1} \ell$, where $\ell=\operatorname{gcd}(n, m)$. Then the polynomial

$$
g(x)=x^{n}+\varepsilon x^{m}+\delta, \quad \varepsilon, \delta \in\{1,-1\},
$$

is irreducible unless $n_{1}+m_{1} \equiv 0(\bmod 3)$ and one of the following three conditions holds:

(1) $n_{1}, m_{1}$ are both odd and $\varepsilon=1$.

(2) $n_{1}$ is even and $\delta=1$.

(3) $m_{1}$ is even and $\varepsilon=\delta$.

In any of these exceptional cases, $g(x)$ is the product of the polynomial

$$
x^{2 \ell}+\varepsilon^{m_{1}} \delta^{n_{1}} x^{\ell}+1
$$

and a second irreducible polynomial. 
To prove Theorem 1.4 we will need to examine the conditions for $r_{n, m}=r_{q, p}$. Clearly, if one of $n, m$ is equal to one of $p, q$, then the other must be equal as well. Without loss of generality we assume that $n>q$. In this case we must have $n>q>p>m$.

Lemma 4.2. Let $n>q>p>m$ be positive integers with $\operatorname{gcd}(n, m, q, p)=1$. Then $r_{n, m}=r_{q, p}$ if and only if $(n, m, q, p)=(5,1,3,2)$.

Proof. It is easy to check that if $(n, m, q, p)=(5,1,3,2)$, then $r_{n, m}=r_{q, p}$ because

$$
x^{5}+x-1=\left(x^{3}+x^{2}-1\right)\left(x^{2}-x+1\right) .
$$

The other direction is more involved. We consider several cases and apply Proposition 4.1. Let $f(x)=x^{n}+x^{m}-1$ and $g(x)=x^{q}+x^{p}-1$. Assume that $r_{n, m}=r_{q, p}$. Then $f(x)$ must be reducible. By Proposition 4.1, if $n \geq 2 m$, then $f(x)=\left(x^{2 \ell} \pm x^{\ell}+1\right) h_{1}(x)$, where $h_{1}(x)$ is irreducible and $\ell=\operatorname{gcd}(n, m)$. If $n<2 m$ we may consider the polynomial $-x^{n} f\left(x^{-1}\right)=x^{n}-x^{n-m}-1$, which is reducible and thus has the form $-x^{n} f\left(x^{-1}\right)=\left(x^{2 \ell} \pm x^{\ell}+1\right) h_{2}(x)$ so that $f\left(x^{-1}\right)=\left(1 \pm x^{-\ell}+x^{-2 \ell}\right)\left(-x^{-(n-2 \ell)} h_{2}(x)\right)$. In both cases we obtain

$$
f(x)=\left(x^{2 \ell} \pm x^{\ell}+1\right) h(x),
$$

where $h(x)$ is irreducible by Proposition 4.1. Since all roots of $x^{2 \ell} \pm x^{\ell}+1$ are on the unit circle, we know that $h\left(r_{n, m}\right)=0$. It follows that $h(x) \mid g(x)$. We now consider two cases.

Case 1. Assume that $g(x)$ is irreducible so that $h(x)=g(x)$. We have

$$
\begin{aligned}
x^{n}+x^{m}-1 & =\left(x^{2 \ell}+\varepsilon x^{\ell}+1\right)\left(x^{q}+x^{p}-1\right) \\
& =x^{q+2 \ell}+x^{p+2 \ell}-x^{2 \ell}+\varepsilon x^{q+\ell}+\varepsilon x^{p+\ell}-\varepsilon x^{\ell}+x^{q}+x^{p}-1,
\end{aligned}
$$

where $\varepsilon \in\{1,-1\}$. It follows that $n=q+2 \ell$ and the middle seven terms on the right hand side must combine to become $x^{m}$. Suppose $\varepsilon=1$; we note that if we set $x=1$, then the two sides are not equal, which is a contradiction. Hence we must have $\varepsilon=-1$. This yields

$$
x^{p+2 \ell}-x^{2 \ell}-x^{q+\ell}-x^{p+\ell}+x^{\ell}+x^{q}+x^{p}=x^{m} .
$$

But $m<p<q$. It follows that $m=\ell, p=2 \ell, q=p+\ell=3 \ell$ and $p+2 \ell=q+\ell$. Now $n=q+2 \ell=5 \ell$. Since $\operatorname{gcd}(n, m, q, p)=1$ we have $\ell=1$ and $(n, m, q, p)=(5,1,3,2)$.

Case 2. Assume that $g(x)$ is reducible. Then as before $g(x)=\left(x^{2 e}+\delta x^{e}+1\right) k(x)$, where $\operatorname{gcd}(q, p)=e, k(x)$ is irreducible and $\delta \in\{1,-1\}$. Since $x^{2 e} \pm x^{e}+1$ has no root in $(0,1)$, so again $k\left(r_{q, p}\right)=0$. It follows from the fact that both $h(x)$ and $k(x)$ are irreducible that $h(x)=k(x)$. Thus

$$
\left(x^{2 e}+\delta x^{e}+1\right)\left(x^{n}+x^{m}-1\right)=\left(x^{2 \ell}+\varepsilon x^{\ell}+1\right)\left(x^{q}+x^{p}-1\right) .
$$

Plug in $x=1$; we easily see that $\varepsilon=\delta$. From $n+2 e=q+2 \ell$ we know that $e<\ell$. In particular, since $\ell=\operatorname{gcd}(n, m)$ we also have $e<m$. But this means the term $-\delta x^{e}$ on the left hand side cannot be cancelled out by any other term on the left hand side. Nor can it be cancelled out by any term on the right hand side because $q>p>m \geq \ell>e$. This is impossible.

We can now complete the proof of Theorem 1.4 . 
Proof of Theorem 1.4. First we prove the if part. It suffices to show that $\mathcal{D}\left(\lambda^{5}, \lambda\right) \sim$ $\mathcal{D}\left(\lambda^{3}, \lambda^{2}\right)$. Note that iterating the $\lambda$ term in $\left(\lambda^{5}, \lambda\right)$ leads to a contraction vector $\left(\lambda^{5}, \lambda^{6}, \lambda^{2}\right)$. Thus $\mathcal{D}\left(\lambda^{5}, \lambda\right) \sim \mathcal{D}\left(\lambda^{5}, \lambda^{6}, \lambda^{2}\right)$. On the other hand, iterating the $\lambda^{3}$ term in $\left(\lambda^{3}, \lambda^{2}\right)$ yields $\left(\lambda^{6}, \lambda^{5}, \lambda^{2}\right)$. Thus $\mathcal{D}\left(\lambda^{3}, \lambda^{2}\right) \sim \mathcal{D}\left(\lambda^{6}, \lambda^{5}, \lambda^{2}\right)$. Clearly $\mathcal{D}\left(\lambda^{5}, \lambda^{6}, \lambda^{2}\right)=\mathcal{D}\left(\lambda^{6}, \lambda^{5}, \lambda^{2}\right)$. Hence $\mathcal{D}\left(\lambda^{5}, \lambda\right) \sim \mathcal{D}\left(\lambda^{3}, \lambda^{2}\right)$.

Now we prove the only if part. Assume that $\operatorname{dim}_{H} E=\operatorname{dim}_{H} F$ and $\left(\rho_{1}, \rho_{2}\right) \neq$ $\left(\tau_{1}, \tau_{2}\right)$. We will show that the condition (2) in Theorem 1.4 must hold. Let $c=\operatorname{rank}\left(\rho_{1}, \rho_{2}\right)$. If $c=2$, then $\left(\tau_{1}, \tau_{2}\right)$ must be a permutation of $\left(\rho_{1}, \rho_{2}\right)$ by Theorem [1.3. This yields $\left(\rho_{1}, \rho_{2}\right)=\left(\tau_{1}, \tau_{2}\right)$, a contradiction. So we must have $\operatorname{rank}\left\langle\rho_{1}, \rho_{2}, \tau_{1}, \tau_{2}\right\rangle=1$, and thus there exists a $\lambda \in(0,1)$ such that

$$
\rho_{1}=\lambda^{n}, \rho_{2}=\lambda^{m}, \tau_{1}=\lambda^{q}, \tau_{2}=\lambda^{p}
$$

for some positive integers $n, m, q, p$ with $\operatorname{gcd}(n, m, q, p)=1$.

Let $s$ be the common Hausdorff dimension of $E$ and $F$; then $x^{n}+x^{m}=1$ and $x^{q}+x^{p}=1$ for $x=\lambda^{s}$. Thus, from assumptions $\rho_{1} \leq \rho_{2}, \tau_{1} \leq \tau_{2}, \rho_{1} \leq \tau_{1}$ and $\left(\rho_{1}, \rho_{2}\right) \neq\left(\tau_{1}, \tau_{2}\right)$, we must have $n>p \geq q>m$. Note that if $p=q$, then the roots of $x^{n}+x^{m}-1=0$ are all algebraic integers while $x=\sqrt[q]{1 / 2}$ is not an algebraic integer, which is a contradiction. Thus we have $n>q>p>m$. It follows from Lemma 4.2 that $(n, m, q, p)=(5,1,3,2)$ so that condition (2) holds. This proves the theorem.

\section{TheOREM 1.5] AND SOME OTHER RESUlts}

In the study of self-similar sets it is useful to consider the symbolic spaces. For any $m \geq 1$ let $\Sigma_{m}$ denote the set of all words $\mathbf{w}=i_{1} i_{2} i_{3} \cdots$ with infinite length, where each $i_{j} \in\{1,2, \ldots, m\}$. For such a $\mathbf{w} \in \Sigma_{m}$ we use the notation $\mathbf{w}(k)=i_{k}$ and $[\mathbf{w}]_{k}=i_{1} i_{2} \cdots i_{k}$. For any $\boldsymbol{\rho}=\left(\rho_{1}, \rho_{2}, \ldots, \rho_{m}\right), 0<\rho_{j}<1$, we can define a metric $\mathbf{d}_{\boldsymbol{\rho}}(.,$.$) on \Sigma_{m}$ as follows: Let $\mathbf{z}, \mathbf{w} \in \Sigma_{m}^{*}$. If $\mathbf{z}(1) \neq \mathbf{w}(1)$, then set $\mathbf{d}_{\boldsymbol{\rho}}(\mathbf{z}, \mathbf{w})=1$; otherwise set $\mathbf{d}_{\boldsymbol{\rho}}(\mathbf{z}, \mathbf{w})=\rho_{[\mathbf{z}]_{k}}$, where $[\mathbf{z}]_{k}=[\mathbf{w}]_{k}$ but $\mathbf{z}(k+1) \neq \mathbf{w}(k+1)$, and $\rho_{[\mathbf{z}]_{k}}:=\prod_{j=1}^{k} \rho_{\mathbf{z}(j)}$. It is well known that $\mathbf{d}_{\boldsymbol{\rho}}$ is indeed a metric on $\Sigma_{m}$. We shall denote the metric space $\Sigma_{m}$ associate with this metric by $\left(\Sigma_{m}, \mathbf{d}_{\boldsymbol{\rho}}\right)$.

Lemma 5.1. Let $\boldsymbol{\rho}=\left(\rho_{1}, \ldots, \rho_{m}\right)$ be a contraction vector and $E \in \mathcal{D}(\boldsymbol{\rho})$. Then there exists a bi-Lipschitz map from $\left(\Sigma_{m}, \mathbf{d}_{\boldsymbol{\rho}}\right)$ to $E$.

Proof. Assume that $E$ is the attractor of the IFS $\left\{\phi_{j}\right\}_{j=1}^{m}$ where the contraction ratio of $\phi_{j}$ is $\rho_{j}$. Fix some $a \in E$. Since the IFS satisfies the strong open set condition, each $x \in E$ has a unique representation $x=\phi_{\mathbf{w}}(a)$, where $\mathbf{w}=i_{1} i_{2} \cdots \in$ $\Sigma_{m}$, using the standard notation $\phi_{\mathbf{w}}(a):=\lim _{k \rightarrow \infty} \phi_{i_{1}} \circ \phi_{i_{2}} \circ \cdots \circ \phi_{i_{k}}(a)$. Let $C_{1}$ denote the smallest distances among the sets $\left\{\phi_{j}(E)\right\}_{j=1}^{m}$. Let $C_{2}$ denote the diameter of $E$.

Now define $f:\left(\Sigma_{m}, \mathbf{d}_{\rho}\right) \longrightarrow E$ by $f(\mathbf{w})=\phi_{\mathbf{w}}(a)$. Note that $E$ is dust-like so that

$$
C_{1} \mathbf{d}_{\boldsymbol{\rho}}(\mathbf{w}, \mathbf{z}) \leq\left|\phi_{\mathbf{w}}(a)-\phi_{\mathbf{z}}(a)\right| \leq C_{2} \mathbf{d}_{\boldsymbol{\rho}}(\mathbf{w}, \mathbf{z}) .
$$

It follows that $f$ is a bi-Lipschitz map from $\left(\Sigma_{m}, \mathbf{d}_{\rho}\right)$ to $E$.

Theorem 5.2. Assume that $\mathcal{D}\left(\rho_{1}, \ldots, \rho_{m}\right)$ and $\mathcal{D}\left(\tau_{1}, \ldots, \tau_{n}\right)$ are Lipschitz equivalent. Let $s=\operatorname{dim}_{H} \mathcal{D}\left(\rho_{1}, \ldots, \rho_{m}\right)$. Then for any $r>s, \mathcal{D}\left(\rho_{1}^{r}, \ldots, \rho_{m}^{r}\right)$ and $\mathcal{D}\left(\tau_{1}^{r}, \ldots, \tau_{n}^{r}\right)$ are also Lipschitz equivalent. 
Proof. Let $\boldsymbol{\rho}^{r}=\left(\rho_{1}^{r}, \ldots, \rho_{m}^{r}\right)$ and $\boldsymbol{\tau}^{r}=\left(\tau_{1}^{r}, \ldots, \tau_{n}^{r}\right)$. By Lemma 5.1] it suffices to establish the Lipschitz equivalence of $\left(\Sigma_{m}, \mathbf{d}_{\boldsymbol{\rho}^{r}}\right)$ and $\left(\Sigma_{n}, \mathbf{d}_{\boldsymbol{\tau}^{r}}\right)$. Since $\mathcal{D}(\boldsymbol{\rho})$ is Lipschitz equivalent to $\mathcal{D}(\boldsymbol{\tau})$, there is a bi-Lipschitz map $f:\left(\Sigma_{m}, \mathbf{d}_{\boldsymbol{\rho}}\right) \longrightarrow\left(\Sigma_{n}, \mathbf{d}_{\boldsymbol{\tau}}\right)$ with

$$
C^{\prime} \mathbf{d}_{\boldsymbol{\rho}}(\mathbf{z}, \mathbf{w}) \leq \mathbf{d}_{\boldsymbol{\tau}}(f(\mathbf{z}), f(\mathbf{w})) \leq C \mathbf{d}_{\boldsymbol{\rho}}(\mathbf{z}, \mathbf{w})
$$

for all $\mathbf{w}, \mathbf{z} \in\left(\Sigma_{m}, \mathbf{d}_{\boldsymbol{\rho}}\right)$, where $C, C^{\prime}>0$.

Observe that since $r>\operatorname{dim}_{H}(\mathcal{D}(\boldsymbol{\rho}))$, we have $\sum_{j=1}^{m} \rho_{j}^{r}<1$. This implies that $\mathcal{D}\left(\boldsymbol{\rho}^{r}\right)$ is non-empty, as is $\mathcal{D}\left(\boldsymbol{\tau}^{r}\right)$ by the same token. Now $f$ can be viewed as a map from $\left(\Sigma_{m}, \mathbf{d}_{\boldsymbol{\rho}^{r}}\right)$ to $\left(\Sigma_{n}, \mathbf{d}_{\boldsymbol{\tau}^{r}}\right)$. We show that it is bi-Lipschitz. Note that we have

$$
\mathbf{d}_{\boldsymbol{\rho}^{r}}=\mathbf{d}_{\boldsymbol{\rho}}^{r}, \quad \mathbf{d}_{\boldsymbol{\tau}^{r}}=\mathbf{d}_{\boldsymbol{\tau}}^{r} .
$$

Thus the inequalities (5.2) hold for $\mathbf{d}_{\boldsymbol{\rho}^{r}}$ and $\mathbf{d}_{\boldsymbol{\tau}^{r}}$, with constants $C^{r}$ and $C^{\prime r}$. The Lipschitz equivalence now follows immediately.

We now consider another kind of Lipschitz equivalence. Let $\boldsymbol{\rho}=\left(\rho_{1}, \ldots, \rho_{m}\right)$ and $\boldsymbol{\tau}=\left(\tau_{1}, \ldots, \tau_{n}\right)$ be two contraction vectors. It is clear that if $\left(\tau_{1}, \ldots, \tau_{m}\right)$ is a permutation of $\left(\rho_{1}, \ldots, \rho_{m}\right)$, then $\mathcal{D}(\boldsymbol{\rho})=\mathcal{D}(\boldsymbol{\tau})$. So we may without of loss generality from now on assume that all contraction ratios $\boldsymbol{\rho}=\left(\rho_{1}, \ldots, \rho_{m}\right)$ are in the standard form in the sense that $0<\rho_{1} \leq \rho_{2} \leq \cdots \leq \rho_{m}<1$. Let $\Phi:=\left\{\phi_{j}\right\}_{j=1}^{m}$ be an IFS with contraction ratios $\boldsymbol{\rho}=\left(\rho_{j}\right)$ that satisfies the SSC. The attractor $E$ of $\Phi$ is the unique compact set satisfying $E=\bigcup_{j=1}^{m} \phi_{j}(E)$. With the SSC all $\left\{\phi_{j}(E)\right\}_{j=1}^{m}$ are disjoint. We say that an IFS $\Psi=\left\{\psi_{i}\right\}_{i=1}^{n}$ is derived from $\Phi$ if $\Psi(E)=E$, all $\left\{\psi_{i}(E)\right\}$ are disjoint, and each $\psi_{i}$ has the form

$$
\psi_{i}(x)=\phi_{j_{1}} \circ \phi_{j_{2}} \circ \cdots \circ \phi_{j_{k}}(x)
$$

for some $1 \leq j_{1}, j_{2}, \ldots, j_{k} \leq m$.

Definition 5.3. Let $\boldsymbol{\rho}$ and $\boldsymbol{\tau}$ be two contraction vectors. We say $\boldsymbol{\tau}$ is dervied from $\boldsymbol{\rho}$ if there is an IFS $\Phi=\left\{\phi_{j}\right\}_{j=1}^{m}$ with contraction vector $\boldsymbol{\rho}$ satisfying the SSC and another IFS $\Psi=\left\{\psi_{i}\right\}_{i=1}^{n}$ with contraction vector $\boldsymbol{\tau}$ such that $\Psi$ is derived from $\Phi$. We say $\boldsymbol{\rho}$ and $\boldsymbol{\tau}$ are equivalent, and denoted it by $\boldsymbol{\rho} \sim \boldsymbol{\tau}$, if there exists a sequence

$$
\boldsymbol{\rho}=\boldsymbol{\rho}_{1}, \boldsymbol{\rho}_{2}, \ldots, \boldsymbol{\rho}_{N}=\boldsymbol{\tau}
$$

such that $\boldsymbol{\rho}_{j+1}$ is derived from $\boldsymbol{\rho}_{j}$ or vice versa for $1 \leq j<N$.

Lemma 5.4. Assume that $\boldsymbol{\rho}$ is equivalent to $\boldsymbol{\tau}$. Then $\mathcal{D}(\boldsymbol{\rho}) \sim \mathcal{D}(\boldsymbol{\tau})$.

Proof. By definition there exists a sequence

$$
\rho=\rho_{1}, \rho_{2}, \ldots, \rho_{N}=\tau
$$

such that $\boldsymbol{\rho}_{j+1}$ is derived from $\boldsymbol{\rho}_{j}$ or vice versa for any $1 \leq j<N$. We only need to prove that $\mathcal{D}\left(\boldsymbol{\rho}_{j}\right) \sim \mathcal{D}\left(\boldsymbol{\rho}_{j+1}\right)$. To this end we may assume without loss of generality that $\boldsymbol{\tau}$ is derived from $\boldsymbol{\rho}$, and we prove that $\mathcal{D}(\boldsymbol{\rho}) \sim \mathcal{D}(\boldsymbol{\tau})$. But by definition there exist IFSs $\Phi$ and $\Psi$ with contraction ratios $\boldsymbol{\rho}$ and $\boldsymbol{\tau}$, respectively, satisfying the SSC such that $\Psi$ is derived from $\Phi$. Thus they have the same attractor, and hence $\mathcal{D}(\boldsymbol{\rho}) \sim \mathcal{D}(\boldsymbol{\tau})$.

Remark. Note that it is possible that $\boldsymbol{\rho} \sim \boldsymbol{\tau}$, but one is not derived from another. One such example is $\boldsymbol{\rho}=\left(\rho^{5}, \rho\right)$ and $\boldsymbol{\tau}=\left(\rho^{3}, \rho^{2}\right)$. Observe that $\left(\rho^{6}, \rho^{5}, \rho^{2}\right)$ is derived both from $\rho$ and from $\tau$. Thus $\rho \sim \tau$. However, neither is derived from 
the other. In fact, it is possible to show that there exists no dust-like self-similar set that is the attractor of both $\Phi$ with contraction ratios $\rho$ and $\Psi$ with contraction ratios $\tau$.

Proof of Theorem 1.5. Assume that $\mathcal{D}(\boldsymbol{\rho}) \sim \mathcal{D}(\boldsymbol{\tau})$. We prove (1) and (2). Condition (1) is obvious because the two classes of sets have the same Hausdorff dimension, which is $\log m / \log \left(\rho^{-1}\right)$. We now prove (2). By Theorem 1.2 there exists some $q \in \mathbb{Z}^{+}$such that

$$
\operatorname{sgp}\left(\tau_{1}^{q}, \ldots, \tau_{n}^{q}\right) \subset \operatorname{sgp}\left(\rho_{1}, \ldots, \rho_{m}\right)=\left\{1, \rho, \rho^{2}, \ldots\right\} .
$$

Thus each $\tau_{j}^{q}=\rho^{p_{j}}$ for some $p_{j} \in \mathbb{N}$, and hence $\tau_{j}=\rho^{p_{j} / q}$. We may without loss of generality assume that $q$ is coprime with $\operatorname{gcd}\left(p_{1}, \ldots, p_{n}\right)$.

Now $m \rho^{s}=1$ and $\rho^{s}=1 / m$ so that $\mathbb{Q}\left(\tau_{1}^{s}, \ldots, \tau_{n}^{s}\right)=\mathbb{Q}\left(\rho^{s}\right)=\mathbb{Q}$. It follows that each $\tau_{j}^{s} \in \mathbb{Q}$. Thus $m^{p_{j} / q} \in \mathbb{Q}$. But $m$ is an integer, so we must have $m^{p_{j} / q} \in \mathbb{Z}$. Combining this with $\operatorname{gcd}\left(q, p_{1}, \ldots, p_{n}\right)=1$, we have $m^{1 / q} \in \mathbb{Z}$. Finally, $\tau_{j}=\rho^{p_{j} / q}$ so that $\log \tau_{j} / \log \rho \in \frac{1}{q} \mathbb{Z}$.

Conversely, assume that conditions (1) and (2) hold. Define $\lambda=\rho^{1 / q}$. Given $j=1, \ldots, n$, we know from $\log \tau_{j} / \log \rho \in \frac{1}{q} \mathbb{Z}^{+}$that $\log \tau_{j} / \log \lambda \in \mathbb{Z}^{+}$, and hence $\tau_{j}=\lambda^{p_{j}}$ for some $p_{j} \in \mathbb{Z}^{+}$. We prove $\mathcal{D}(\boldsymbol{\rho}) \sim \mathcal{D}(\boldsymbol{\tau})$ by showing that $\boldsymbol{\rho} \sim \boldsymbol{\tau}$.

Define $k=m^{1 / q}$. Write $\boldsymbol{\lambda}=(\lambda, \ldots, \lambda) \in \mathbb{R}^{k}$. Note that $k \lambda^{s}=1$ because $\left(k \lambda^{s}\right)^{q}=m \rho^{s}=1$. With $0<s<1$ we know that there exists an IFS $\Phi=\left\{\phi_{j}\right\}_{j=1}^{k}$ with the SSC and contraction vector $\boldsymbol{\lambda}$. We introduce the following notation. Let $r$ be any given positive integer. For any $\mathbf{j}=j_{1} j_{2} \cdots j_{r} \in\{1,2, \ldots, k\}^{r}$ we shall use $\phi_{\mathbf{j}}$ to denote the map $\phi_{\mathbf{j}}=\phi_{j_{1}} \circ \phi_{j_{2}} \circ \cdots \circ \phi_{j_{r}}$. Denote by $\Phi^{r}$ the IFS $\Phi^{r}=$ $\left\{\phi_{\mathbf{j}}: \mathbf{j} \in\{1,2, \ldots, k\}^{r}\right\}$. Clearly $\Phi^{r}$ is an iterate of $\Phi$, and it has a contraction vector $\left(\lambda^{r}, \lambda^{r}, \ldots, \lambda^{r}\right) \in \mathbb{R}^{k^{r}}$. Thus letting $r=q$ we see that $\boldsymbol{\rho}$ is derived from $\boldsymbol{\lambda}$ and hence $\boldsymbol{\lambda} \sim \boldsymbol{\rho}$. We prove that $\boldsymbol{\lambda} \sim \boldsymbol{\tau}$ also.

Without loss of generality we assume that $p_{1} \leq p_{2} \leq \cdots \leq p_{n}$. We show that there exists an iterate $\Psi$ of $\Phi$ such that the contraction ratios of $\Psi$ are given by $\tau$. This can be proved by selectively iterating the maps in $\Phi$. First set

$$
\Phi_{1}:=\Phi^{p_{1}}=\left\{\phi_{\mathbf{j}}: \mathbf{j} \in\{1,2, \ldots, k\}^{p_{1}}\right\} .
$$

Note that all $\phi_{\mathbf{j}}$ in $\Phi_{1}$ have contraction ratio $\lambda^{p_{1}}$. Next we leave one of the maps in $\Phi_{1}$, say, $\phi_{\mathbf{j}_{1}}$, intact and iterate the rest of the maps as follows: We replace each $\phi_{\mathbf{j}}$ where $\mathbf{j} \neq \mathbf{j}_{1}$ by the maps $\phi_{\mathbf{j}} \circ \phi_{\mathbf{i}}, \mathbf{i} \in\{1, \ldots, k\}^{p_{2}-p_{1}}$. (Here if $p_{2}=p_{1}$ we do nothing.) This leads to another IFS $\Phi_{2}$ that is an iterate of $\Phi_{1}$, and it has the property that with the exception of the one map $\phi_{\mathbf{j}_{1}}$ all other maps in it have a contraction ration $\lambda^{p_{2}}$. We select one of them and label it $\phi_{\mathbf{j}_{2}}$.

This process is now continued further. For each $\phi_{\mathbf{j}}$ in $\Phi_{2}$ that is not $\phi_{\mathbf{j}_{1}}$ and $\phi_{\mathbf{j}_{2}}$, we iterate it by replacing $\phi_{\mathbf{j}}$ with the maps $\phi_{\mathbf{j}} \circ \phi_{\mathbf{i}}, \mathbf{i} \in\{1, \ldots, k\}^{p_{3}-p_{2}}$. (Again if $p_{3}=p_{2}$ we do nothing.) These iterations lead to the IFS $\Phi_{3}$, where with the exception of the maps $\phi_{\mathbf{j}_{1}}$ and $\phi_{\mathbf{j}_{2}}$ all other maps have contraction ratios $\lambda^{p_{3}}$. We select one of them and label it $\phi_{\mathbf{j}_{3}}$. Continuing this process we eventually obtain an IFS $\Phi_{L}=\left\{\phi_{\mathbf{j}_{1}}, \phi_{\mathbf{j}_{2}}, \ldots, \phi_{\mathbf{j}_{L}}\right\}$.

Finally, we show that $L=n$. If $L<n$, then the contraction ratios of $\Phi_{L}$ are $\left(\tau_{j}\right) \in \mathbb{R}^{L}$. But the attractor of $\Phi_{L}$ is the same as the attractor of $\Phi$, which has Hausdorff dimension $s$. Thus $\sum_{j=1}^{L} \tau_{j}^{s}=1$, but this contradicts $\sum_{j=1}^{L} \tau_{j}^{n}=1$. Thus $L \geq n$. By the same argument we cannot have $L>n$. Hence $L=n$. It follows that 
the contraction ratios of $\Phi_{L}$ are given by $\tau$. This $\tau$ is derived from $\boldsymbol{\lambda}$, and hence $\boldsymbol{\tau} \sim \boldsymbol{\lambda}$. It follows that $\boldsymbol{\rho} \sim \boldsymbol{\tau}$. The theorem is thus proved.

\section{Appendix A. The proof of Lemma 2.7}

Proof. Since $F$ is dust-like, $F$ satisfies the open set condition, i.e., there exists an open set $V$ such that $V \supset \cup_{i=1}^{n} \psi_{i}(V)$ and $\psi_{i}(V) \cap \psi_{j}(V)=\emptyset$ for distinct $i, j$. It is clear that there exists a ball $B$ in $V$. Now, given a non-empty set, $A \subset \mathbb{R}^{d}$. Define

$$
\mathcal{I}=\left\{\mathbf{i}: F_{\mathbf{i}} \cap A \neq \emptyset \text { and } c_{1} \operatorname{diam} A \leq \operatorname{diam} F_{\mathbf{i}} \leq c_{2} \operatorname{diam} A\right\} .
$$

Take any $\mathcal{J} \subset \mathcal{I}$ such that $F_{\mathbf{i}} \cap F_{\mathbf{j}}=\emptyset$ for any distinct $\mathbf{i}, \mathbf{j} \in \mathcal{J}$. It suffices to prove that $\operatorname{card}(\mathcal{J})$ is bounded.

For each $\mathbf{i} \in \mathcal{J}$, we define $\delta_{\mathbf{i}}=\operatorname{diam} F_{\mathbf{i}} \cdot \frac{\operatorname{diam} V}{\operatorname{diam} F}$ and $N_{\delta_{\mathbf{i}}}(A)=\{y: d(x, y)<$ $\delta_{\mathbf{i}}$ for some $\left.x \in A\right\}$. Then $N_{\delta_{\mathbf{i}}}(A) \supset \psi_{\mathbf{i}}(V) \supset \psi_{\mathbf{i}}(B)$. Let $\delta=\sup \left\{\delta_{\mathbf{i}}: \mathbf{i} \in \mathcal{J}\right\}$; then $\delta \leq \operatorname{diam} A \cdot \frac{c_{2} \operatorname{diam} V}{\operatorname{diam} F}$ and

$$
N_{\delta}(A) \supset \bigcup_{\mathbf{i} \in \mathcal{J}} \psi_{\mathbf{i}}(B)
$$

We will show that the union on the right hand side is disjoint. Otherwise, assume that $\psi_{\mathbf{i}}(B) \cap \psi_{\mathbf{j}}(B) \neq \emptyset$ for distinct $\mathbf{i}, \mathbf{j} \in \mathcal{J}$. Then $\psi_{\mathbf{i}}(V) \cap \psi_{\mathbf{j}}(V) \neq \emptyset$. By the open set condition, we must have $\psi_{\mathbf{i}}(V) \subset \psi_{\mathbf{j}}(V)$ or $\psi_{\mathbf{i}}(V) \supset \psi_{\mathbf{j}}(V)$. It follows that $F_{\mathbf{i}} \subset F_{\mathbf{j}}$ or $F_{\mathbf{i}} \supset F_{\mathbf{j}}$, which contradicts the mutual disjointness of $F_{\mathbf{i}}$.

Notice that $\psi_{\mathbf{i}}(B)$ is a ball with diameter $\frac{\operatorname{diam} F_{\mathbf{i}} \cdot \operatorname{diam} B}{\operatorname{diam} F} \geq c_{1} \operatorname{diam} A \cdot \frac{\operatorname{diam} B}{\operatorname{diam} F}=$ : $c_{1}^{*} \operatorname{diam} A$ and $N_{\delta}(A)$ is contained in a ball with diameter

$$
2(|A|+\delta) \leq 2 \operatorname{diam} A \cdot\left(1+\frac{c_{2} \operatorname{diam} V}{\operatorname{diam} F}\right)=: c_{2}^{*} \operatorname{diam} A .
$$

Thus $N_{\delta}(A)$ can contain at most $c_{3}:=\left(c_{2}^{*} / c_{1}^{*}\right)^{d}$ mutually disjoint $\psi_{\mathbf{i}}(B)$ so that $\operatorname{card}(\mathcal{J}) \leq c_{3}$.

Notice that $c_{3}=\left(c_{2}^{*} / c_{1}^{*}\right)^{d}$, where $c_{1}^{*}$ and $c_{2}^{*}$ are two positive constants only dependent on $c_{1}, c_{2}$ and the IFS $\left\{\psi_{i}\right\}$. This completes the proof of the lemma.

\section{ACKNOWLEDGEMENTS}

We are grateful to Martin Kassabov and Ravi Ramakrishna for discussions in connection with the material in Section 4 and to Andrzej Schinzel for pointing out the reference [9].

\section{REFERENCES}

1. D. Cooper and T. Pignataro, On the shape of Cantor sets, J. Differential Geom., 28 (1988), 203-221. MR961514 (89k:58160)

2. G. David and S. Semmes, Fractured fractals and broken dreams: Self-similar geometry through metric and measure, Oxford Univ. Press, 1997. MR1616732 (99h:28018)

3. K. J. Falconer, Fractal Geometry: Mathematical Foundations and Applications, New York: John Wiley \& Sons, 1990. MR1102677 (92j:28008)

4. K. J. Falconer, Techniques in fractal geometry, Chichester: John Wiley \& Sons, 1997. MR:1449135 (99f:28013)

5. K. J. Falconer and D. T. Marsh, Classification of quasi-circles by Hausdorff dimension, Nonlinearity, 2 (1989), 489-493. MR.1005062 (91a:58108)

6. K. J. Falconer and D. T. Marsh, On the Lipschitz equivalence of Cantor sets, Mathematika, 39 (1992), 223-233. MR:1203278 (93m:28011)

7. T. W. Hungerford, Algebra, Graduate Texts in Mathematics 73, Springer: New York, 1980. MR600654 (82a:00006) 
8. J. E. Hutchinson, Fractals and self-similarity, Indiana Univ. Math. J., 30 (1981), 713-747. MR625600 (82h:49026)

9. W. Ljunggren, On the irreducibility of certain trinomials and quadrinomials, Math. Scand., 8 (1960), 65-70. MR0124313 (23:A1627)

10. P. Mattila and P. Saaranen, Ahlfors-David regular sets and bilipschitz maps, Ann. Acad. Sci. Fenn. Math., 34 (2009), 487-502. MR2553808

11. H. Rao, H.-J. Ruan and L.-F. Xi, Lipschitz equivalence of self-similar sets, C. R. Acad. Sci. Paris. Ser. I, 342 (2006), 191-196. MR2198192 (2006m:28013)

12. H. Rao, H.-J. Ruan and Y.-M. Yang, Gap sequence, Lipschitz equivalence and box dimension of fractal sets, Nonlinearity, 6 (2008), 1339-1347. MR2422383 (2009f:28020)

13. A. Schief, Separation properties for self-similar sets, Proc. Amer. Math. Soc., 122 (1994), 111-115. MR1191872 (94k:28012)

14. Z.-Y. Wen and L.-F. Xi, Relations among Whitney sets, self-similar arcs and quasi-arcs, Israel J. Math., 136 (2003), 251-267. MR1998112 (2004e:28009)

15. L.-F. Xi, Lipschitz equivalence of dust-like self-similar sets, Math. Z., 266 (2010), 683-691. MR2719426

16. L.-F. Xi, Lipschitz equivalence of self-conformal sets, J. London Math. Soc., 70 (2004), 369382. MR2078899 (2005h:28029)

17. L.-F. Xi and H.-J. Ruan, Lipschitz equivalence of generalized $\{1,3,5\}-\{1,4,5\}$ self-similar sets, Sci. China Ser. A, 50 (2007), 1537-1551. MR2390469 (2009e:28039)

18. L.-F. Xi and H.-J. Ruan, Lipschitz equivalence of self-similar sets satisfying the strong separation condition (in Chinese), Acta Math. Sinica (Chin. Ser.), 51 (2008), 493-500. MR2453758 (2009i:28024)

19. L.-F. Xi, H.-J. Ruan and Q.-L. Guo, Sliding of self-similar sets, Sci. China Ser. A, 50 (2007), 351-360. MR2334554 (2008m:28011)

Department of Mathematics, Hua Zhong Normal University, Wuhan 430079, People's Republic of China

E-mail address: hrao@mail.ccnu.edu.cn

Department of Mathematics, Zhejiang University, Hangzhou 310027, People's Republic of China - and - Department of Mathematics, Cornell University, Ithaca, New York 14853

E-mail address: ruanhj@zju.edu.cn

Department of Mathematics, Michigan State University, East Lansing, Michigan 48824

E-mail address: ywang@math.msu.edu 